\title{
A component model for dynamic correlations*
}

\author{
Riccardo Colacito $^{\dagger} \quad$ Robert F. Engle $^{\ddagger} \quad$ Eric Ghysels $^{\S}$
}

First Draft: April 2006

This Draft: January 31, 2009

\begin{abstract}
The idea of component models for volatility is extended to dynamic correlations. We propose a model of dynamic correlations with a short- and long-run component specification. We call this class of models DCC-MIDAS as the key ingredients are a combination of the Engle (2002) DCC model, the Engle and Lee (1999) component GARCH model to replace the original DCC dynamics with a component specification and the Engle, Ghysels, and Sohn (2006) GARCH-MIDAS component specification that allows us to extract a long-run correlation component via mixed data sampling. We provide a comprehensive econometric analysis of the new class of models, including conditions for positive semi-definiteness, and provide extensive empirical evidence that supports the model specification.
\end{abstract}

*This research is funded in part by the Morgan Stanley, Equity Market Microstructure Research Grant. We also like to thank seminar participants at the Journal of Financial Econometrics Faro conference of Multivariate Volatility Models, the Forecasting in Rio conference, NYU-Stern, Oxford and Ohio State University for comments.

${ }^{\dagger}$ Department of Finance, Kenan-Flagler Business School, University of North Carolina at Chapel Hill, email: riccardo.colacito@unc.edu

${ }^{\ddagger}$ Department of Finance, Stern School of Business, New York University, e-mail: rengle@stern.nyu.edu

${ }^{\S}$ Department of Finance, Kenan-Flagler Business School, and Department of Economics, University of North Carolina at Chapel Hill, email: eghysels@unc.edu 


\section{Introduction}

Component models have been widely used for volatility dynamics. The motivation is typically based on either one of the following two arguments. First, the component structure allows for a parsimonious representation of complex dependence structures. Second, the components are sometimes linked to economic principles, namely the idea that there are different short- and long-run sources that affect volatility. The purpose of this paper is to propose a component model of dynamic correlations with a shortand long-run component specification. ${ }^{1}$ We call this class of models DCC-MIDAS as the key ingredients are a combination of the Engle (2002) DCC model, the Engle and Lee (1999) component GARCH model to replace the original DCC dynamics with a component specification and the Engle, Ghysels, and Sohn (2006) GARCH-MIDAS component specification that allows us to extract a long-run correlation component via mixed data sampling.

We address the specification, estimation and interpretation of correlation models that distinguish short and long run components. We show that the changes in correlations are indeed very different. Dynamic correlations are a natural extension of the GARCH-MIDAS model to Engle (2002) DCC model. The idea captured by the DCCMIDAS model is similar to that underlying GARCH-MIDAS. In the latter case, two components of volatility are extracted, one pertaining to short term fluctuations, the other pertaining to a secular component. In the GARCH-MIDAS the short run component is a GARCH component, based on daily (squared) returns, that moves around a long-run component driven by realized volatilities computed over a monthly, quarterly or bi-annual basis. The MIDAS weighting scheme helps us extract the slowly moving secular component around which daily volatility moves. Engle, Ghysels, and Sohn (2006) explicitly link the extracted MIDAS component to macroeconomic sources. It is the same logic that is applied here to correlations. Namely, the daily dynamics obey a DCC scheme, with the correlations moving around a long run component. Short-lived effects to correlations will be captured by the autoregressive dynamic structure of DCC, with the intercept of the latter being a slowly moving process

\footnotetext{
${ }^{1}$ It should be noted that there have been several prior attempts to think of component models for correlations, see inter alia Karolyi and Stulz (1996). Our approach focuses on autoregressive conditional correlation models.
} 
that reflects the fundamental or long-run causes of time variation in correlation. ${ }^{2}$

To estimate the parameters of the DCC-MIDAS model we follow the two-step procedure of Engle (2002). We start by estimating the parameters of the univariate conditional volatility models. The second step consists of estimating the DCC-MIDAS parameters with the standardized residuals. We also discuss the regularity conditions we need to impose on the MIDAS-filtered long run correlation component as models of correlations are required to yield positive definite matrices.

The paper concludes with an empirical illustration, showing the benefits of the component specification. Empirical specification tests reveal the superior empirical fit, both in- and out-of-sample of the new class of DCC-MIDAS correlation models.

The remainder of the paper is organized as follows. Section 2 introduces the correlation component model and compares the DCC-MIDAS class of models with original DCC models. Section 3 covers regularity conditions and estimation, while section 5 contains the empirical applications. Section 6 concludes the paper.

\section{A new class of component correlation models}

The purpose of this section is to introduce the class of DCC/MIDAS dynamic correlation models. In a first subsection we provide some preliminaries. The second subsection introduces the structure of DCC/MIDAS.

\subsection{Notation and Preliminaries}

Consider a set of $n$ assets and let the vector of returns be denoted as $\mathbf{r}_{t}=\left[r_{1, t}, \ldots, r_{n, t}\right]^{\prime}$. The novelty of our approach consists of describing the dynamics of conditional variances and correlations, where we take into account both short and long run components. The long run component at time $t$ will be a judiciously chosen weighted average of historical correlations. The assumption is that the long run component can be filtered from empirical correlations. Of course, what is critical is the choice of weights,

\footnotetext{
${ }^{2}$ In principle we can link the secular correlation component to macroeconomic sources, very much like Engle, Ghysels, and Sohn (2006) and Schwert (1989), who study long historical time series and link volatility directly to various key macroeconomic time series.
} 
which will be one of the key ingredients of the model specification. To proceed let us assume that the vector of returns $\mathbf{r}_{t}=\left[r_{1, t}, \ldots, r_{n, t}\right]^{\prime}$ follows the process:

$$
\begin{array}{rll}
\mathbf{r}_{t} & \sim_{i . i . d .} & N\left(\boldsymbol{\mu}, \boldsymbol{H}_{t}\right) \\
\boldsymbol{H}_{t} & =\boldsymbol{D}_{t} \boldsymbol{R}_{t} \boldsymbol{D}_{t}
\end{array}
$$

$\boldsymbol{\mu}$ is where the vector of unconditional means, $\boldsymbol{H}_{t}$ is the conditional covariance matrix and $D_{t}$ is a diagonal matrix with standard deviations on the diagonal, and:

$$
\begin{aligned}
\boldsymbol{R}_{t} & =E_{t-1}\left[\boldsymbol{\xi}_{t} \boldsymbol{\xi}_{t}^{\prime}\right] \\
\boldsymbol{\xi}_{t} & =D_{t}^{-1}\left(\mathbf{r}_{t}-\boldsymbol{\mu}\right)
\end{aligned}
$$

Therefore $\mathbf{r}_{t}=\boldsymbol{\mu}+\boldsymbol{H}_{t}^{\frac{1}{2}} \boldsymbol{\xi}_{t}$ with $\boldsymbol{\xi}_{t} \sim_{i . i . d .} N\left(\mathbf{0}, \boldsymbol{I}_{n}\right)$. In this section we introduce a new class of component correlation models. In a first subsection we discuss model specification. The next subsection covers regularity conditions and deals with estimation.

At the outset, it should be noted that component models for correlations also prompt us to think about component models for volatility which feed into the correlation specification. Indeed the decomposition of the conditional covariance matrix $\boldsymbol{H}_{t}=$ $\boldsymbol{D}_{t} \boldsymbol{R}_{t} \boldsymbol{D}_{t}$ appearing in equation (2.1) with $\boldsymbol{D}_{t}$ a diagonal matrix of standard deviations and $\boldsymbol{R}_{t}$ the conditional correlation matrix suggests a two-step model specification (and estimation) strategy. Consequently, we will first specify $\boldsymbol{D}_{t}$ followed by $\boldsymbol{R}_{t}$.

The univariate volatility models build on recent work by Engle and Rangel (2005) and in particular Engle, Ghysels, and Sohn (2006). Both proposed component models for volatility, where long an short run volatility dynamics are separated. Engle and Rangel (2005) introduce a Spline-GARCH model where the daily equity volatility is a product of a slowly varying deterministic component and a mean reverting unit GARCH. Unlike conventional GARCH or stochastic volatility models, this model permits low frequency volatility to change over time. Engle and Rangel (2005) use an exponential spline as a convenient non-negative parameterization. The recent work of Engle, Ghysels, and Sohn (2006) combines insights from Engle and Rangel (2005) with a framework that is suited to combine data that are sampled at different frequencies. The new approach is inspired by the recent work on mixed data sampling, or MIDAS discussed in Ghysels, Santa-Clara, and Valkanov (2005), Ghysels, Santa- 
Clara, and Valkanov (2006), Forsberg and Ghysels (2004), among others. ${ }^{3}$ Engle, Ghysels, and Sohn (2006) replace the spline specification with a MIDAS polynomial.

The new class of models is called GARCH-MIDAS, since it uses a mean reverting unit daily GARCH process, similar to Engle and Rangel (2005), and a MIDAS polynomial which applies to monthly, quarterly, or bi-annual macroeconomic or financial variables. In what follows we will refer to $g_{i}$ and $m_{i}$ as the short and long run variance components respectively for asset $i$. Engle, Ghysels, and Sohn (2006) consider various specifications for $g_{i}$ and we select only a specific one where the long run component is held constant across the days of the month, quarter or half-year. Alternatively, one can specify $m_{i}$ based on rolling samples that change from day to day. The findings in Engle, Ghysels, and Sohn (2006) show that they yield very similar empirical fits - so we opted for the simplest to implement which involves locally constant long run components. We will denote by $N_{v}^{i}$ the number of days that $m_{i}$ is held fixed. The superscript $i$ indicates that this may be asset-specific. The subscript $v$ differentiates it from a similar scheme that will be introduced later for correlations. It will be convenient to introduce two time scales $t$ and $\tau$. In particular, while $g_{i, t}$ moves daily, $m_{i, \tau}$ changes only once every $N_{v}^{i}$ days.

More specifically we assume that for each asset $i=1, \ldots, n$, univariate returns follow the GARCH-MIDAS process:

$$
r_{i, t}=\mu_{i}+\sqrt{m_{i, \tau} \cdot g_{i, t}} \xi_{i, t}, \quad \forall t=\tau N_{v}^{i}, \ldots,(\tau+1) N_{v}^{i}
$$

where $g_{i, t}$ follows a GARCH(1,1) process:

$$
g_{i, t}=\left(1-\alpha_{i}-\beta_{i}\right)+\alpha_{i} \frac{\left(r_{i, t-1}-\mu_{i}\right)^{2}}{m_{i, \tau}}+\beta_{i} g_{i, t-1}
$$

while the MIDAS component $m_{i, \tau}$ is a weighted sum of $L_{v}^{i}$ lags of realized variances

\footnotetext{
${ }^{3}$ In the context of volatility, Ghysels, Santa-Clara, and Valkanov (2005) studied the traditional riskreturn trade-off and used monthly data to proxy expected returns while the variance was estimated using daily squared returns. The idea was carried a step further in Ghysels, Santa-Clara, and Valkanov (2006) and Forsberg and Ghysels (2004), both papers focusing on predicting volatility at various horizons with high frequency financial data using MIDAS.
} 
$(R V)$ over a long horizon:

$$
m_{i, \tau}=\bar{m}_{i}+\theta_{i} \sum_{l=1}^{L_{v}^{i}} \varphi_{l}\left(\omega_{v}^{i}\right) R V_{i, \tau-l}
$$

where the realized variances involve $N_{v}^{i}$ daily squared returns, namely:

$$
R V_{i, \tau}=\sum_{j=(\tau-1) N_{v}^{i}+1}^{\tau N_{v}^{i}}\left(r_{i, j}\right)^{2} .
$$

Note that $N_{v}^{i}$ could for example be a quarter or a month. The above specification corresponds to the block sampling scheme as defined in Engle, Ghysels, and Sohn (2006), involving so called Beta weights defined as:

$$
\varphi_{l}\left(\omega_{v}^{i}\right)=\frac{\left(1-\frac{l}{L_{v}^{i}}\right)^{\omega_{v}^{i}-1}}{\sum_{j=1}^{L_{v}^{i}}\left(1-\frac{j}{L_{v}^{i}}\right)^{\omega_{v}^{i}-1}}
$$

In practice we will consider cases where the parameters $N_{v}^{i}$ and $L_{v}^{i}$ are independent of $i$, i.e. the same across all series. Similarly, we can also allow for different decay patterns $\omega_{v}^{i}$ across various series, but once again we will focus on cases with common $\omega_{v}$ (see the next subsection for further discussion). Obviously, despite the common parameter specification, we expect that the $m_{i, \tau}$ substantially differ across series, as they are data-driven.

\subsection{The class of DCC/MIDAS dynamic correlation models}

Dynamic correlations are a natural extension of the GARCH-MIDAS model to the Engle (2002) DCC model. More specifically, we will introduce two components, a longrun and short-run one. In the case of volatility we noted that $m_{i, \tau}$ can be formulated either via keeping it locally constant, or else based on a local moving window. Engle, Ghysels, and Sohn (2006) find for volatility that the difference between the two - as noted before. For correlations, we have potentially the same choice. Since the trailing local specification is more general, we adopt this for our formulation. Namely, using 
the standardized residuals $\xi_{i, t}$ it is possible to obtain a matrix $\boldsymbol{Q}_{t}$ whose elements are:

$$
\begin{aligned}
q_{i, j, t} & =\bar{\rho}_{i, j, t}(1-a-b)+a \xi_{i, t-1} \xi_{j, t-1}+b q_{i, j, t-1} \\
\bar{\rho}_{i, j, t} & =\sum_{l=1}^{L_{c}^{i j}} \varphi_{l}\left(\omega_{r}^{i j}\right) c_{i, j, t-l} \\
c_{i, j, t} & =\frac{\sum_{k=t-N_{c}^{i j}}^{t} \xi_{i, k} \xi_{j, k}}{\sqrt{\sum_{k=t-N_{c}^{i j}}^{t} \xi_{i, k}^{2}} \sqrt{\sum_{k=t-N_{c}}^{t} \xi_{j, k}^{2}}}
\end{aligned}
$$

where the weighting scheme is similar to that appearing in (2.5). Note that in the above formulation of $c_{i, j, t}$ we could have used simple cross-products of $\xi_{i, t}$. The normalization will allow us later to discuss regularity conditions in terms of correlation matrices. Correlations can then be computed as:

$$
\rho_{i, j, t}=\frac{q_{i, j, t}}{\sqrt{q_{i, i, t}} \sqrt{q_{j, j, t}}}
$$

We regard $q_{i, j, t}$ as the short run correlation between assets $i$ and $j$, whereas $\bar{\rho}_{i, j, t}$ is a slowly moving long run correlation. Rewriting the first equation of system (2.7) as

$$
q_{i, j, t}-\bar{\rho}_{i, j, t}=a\left(\xi_{i, t-1} \xi_{j, t-1}-\bar{\rho}_{i, j, t}\right)+b\left(q_{i, j, t-1}-\bar{\rho}_{i, j, t}\right)
$$

conveys the idea of short run fluctuations around a time varying long run relationship. The idea captured by the DCC-MIDAS model is similar to that underlying GARCH-MIDAS. In the latter case, two components of volatility are extracted, one pertaining to short term fluctuations, the other pertaining to a secular component. In the GARCH-MIDAS the short run component is a GARCH component, based on daily (squared) returns, that moves around a long-run component driven by realized volatilities computed over a monthly, quarterly or bi-annual basis. The MIDAS weighting scheme helps us extracting the slowly moving secular component around which daily volatility moves. Engle, Ghysels, and Sohn (2006) explicitly link the extracted MIDAS component to macroeconomic sources. It is the same logic that is applied here to correlations. Namely, the daily dynamics obey a DCC scheme, with the correlations moving around a long run component. Short-lived effects on correlations will be captured by the autoregressive dynamic structure of DCC, with the intercept of the latter being a slowly moving process that reflects the fundamental or secular causes of time 
variation in correlation. In principle we can link the long run correlation component to macroeconomic sources, very much like Engle, Ghysels, and Sohn (2006) study long historical time series, similar to Schwert (1989) and link volatility directly to various key macroeconomic time series. ${ }^{4}$ Note that in equation (2.5) we can allow for different weighting schemes across series. Likewise, the specification in (2.7) can potentially accommodate weights $\omega_{r}^{i j}$, lag lengths $N_{c}^{i j}$ and span lengths of historical correlations $L_{c}^{i j}$ to differ across any pair of series. ${ }^{5}$ Typically we will use a single setting common to all pairs of series, similar to the choice of a common MIDAS filter in the in the univariate models. We will discuss in the next subsection the implications of a single versus multiple parameter choices for the DCC-MIDAS filtering scheme.

It is also worth noting that our DCC-MIDAS model shares features with a local dynamic conditional correlation (LDCC) model introduced in Feng (2007), where variances are decomposed into a conditional and a local (unconditional) parts. The correlation structure is modeled by a multivariate nonparametric ARCH-type approach that accommodates the presence of regressors.

To conclude this subsection we fix some notation that will allow us to discuss the general model specification. First, we will collect all the elements $\omega_{r}^{i j}$ into a vector $\underline{\omega}_{r}$, keeping in mind that it may only contain a single element if all weights are equal and we denote $N_{c}=\max _{i, j} N_{C}^{i j}$. We then can write and collect the set of correlations appearing in equation (2.7) yielding generically in matrix form:

$$
\begin{aligned}
\boldsymbol{R}_{t} & =\left(\boldsymbol{Q}_{t}^{*}\right)^{-1 / 2} \boldsymbol{Q}_{t}\left(\boldsymbol{Q}_{t}^{*}\right)^{-1 / 2} \\
\boldsymbol{Q}_{t}^{*} & =\operatorname{diag} \boldsymbol{Q}_{t} \\
\boldsymbol{Q}_{t} & =(1-a-b) \overline{\boldsymbol{R}}_{t}\left(\underline{\omega}_{r}\right)+a \boldsymbol{\xi}_{t} \boldsymbol{\xi}_{t}^{\prime}+b \boldsymbol{Q}_{t-1}
\end{aligned}
$$

\footnotetext{
${ }^{4}$ We prefer not to do this in the current paper, because it would require selecting an ad hoc function to link macro variables to correlations. This function should satisfy the restrictions that correlations are bounded between -1 and 1 and the resulting correlation matrix must be positive semi-definite.

${ }^{5}$ Note that $\left(\omega_{r}^{i j}, N_{c}^{i j}, L_{c}^{i j}\right)=\left(\omega_{r}^{j i}, N_{c}^{j i}, L_{c}^{j i}\right)$ are identical for all $i$ and $j$.
} 


$$
\begin{aligned}
\overline{\boldsymbol{R}}_{t}\left(\underline{\omega}_{r}\right) & =\sum_{l=1}^{L_{c}} \boldsymbol{\Phi}_{l}\left(\underline{\omega}_{r}\right) \odot \boldsymbol{C}_{t-l} \\
\boldsymbol{C}_{t} & =\left(\begin{array}{ccc}
v_{1, t} & 0 & 0 \\
\vdots & \ddots & 0 \\
0 & \cdots & v_{n, t}
\end{array}\right)^{-\frac{1}{2}}\left(\sum_{k=t-N_{c}}^{t} \boldsymbol{\xi}_{k} \boldsymbol{\xi}_{k}^{\prime}\right)\left(\begin{array}{ccc}
v_{1, t} & 0 & 0 \\
\vdots & \ddots & 0 \\
0 & \cdots & v_{n, t}
\end{array}\right)^{-\frac{1}{2}} \\
v_{i, t} & =\sum_{k=t-N_{c}}^{t} \boldsymbol{\xi}_{i, k}^{2}, \quad \forall i=1, \ldots, n
\end{aligned}
$$

where $\Phi_{l}\left(\underline{\omega}_{r}\right)=\varphi_{l}\left(\underline{\omega}_{r}\right) \iota^{\prime}$ and $\odot$ stands for the Hadamard product. ${ }^{6}$

\section{Estimation}

To estimate the parameters of the DCC-MIDAS model we follow the two-step procedure of Engle (2002). We start by collecting the parameters of the univariate conditional volatility models into a vector $\Psi \equiv\left[\left(\alpha_{i}, \beta_{i}, \omega_{i}, m_{i}, \theta_{i}\right), i=1, \ldots, n\right]$. and the parameters of the conditional correlation model into $\Xi \equiv\left(a, b, \underline{\omega}_{r}\right)$. Then the (quasi)likelihood function $Q L$ can be written as:

$$
\begin{aligned}
Q L(\Psi, \Xi) & =Q L_{1}(\Psi)+Q L_{2}(\Psi, \Xi) \\
& \equiv-\sum_{t=1}^{T}\left(n \log (2 \pi)+2 \log \left|\boldsymbol{D}_{t}\right|+\mathbf{r}_{t}^{\prime} \boldsymbol{D}_{t}^{-2} \mathbf{r}_{t}\right)-\sum_{t=1}^{T}\left(\log \left|\boldsymbol{R}_{t}\right|+\boldsymbol{\xi}_{t}^{\prime} \boldsymbol{R}_{t}^{-1} \boldsymbol{\xi}_{t}+\boldsymbol{\xi}_{t}^{\prime} \boldsymbol{\xi}_{t}\right)
\end{aligned}
$$

Given the structure of the log likelihood function, namely the separation of $Q L(\Psi, \Xi)$ into $Q L_{1}(\Psi)$ and $Q L_{2}(\Psi, \Xi)$, we can first estimate the parameters of the univariate GARCH-MIDAS processes, i.e. the parameters in $\Psi$, using $Q L_{1}(\Psi)$ and therefore each single series separately - yielding $\hat{\Psi}$. The second step consists of estimating the DCCMIDAS parameters with the standardized residuals $\hat{\boldsymbol{\xi}}_{t}=\hat{\boldsymbol{D}}_{t}^{-1}\left(\mathbf{r}_{t}-\hat{\boldsymbol{\mu}}\right)$ using $Q L_{2}(\hat{\Psi}, \Xi)$. The estimation of the MIDAS polynomial parameters in the dynamic correlations require some further discussion. The approach we adopt is inspired by the estimation of MIDAS polynomial parameters in the GARCH-MIDAS model.

\footnotetext{
${ }^{6}$ In a later section we will generalize this setup to allow for multiple MIDAS filters in the long-run dynamics of correlations.
} 
So far we were not very explicit about the choice of the polynomial characteristics $L_{v}^{i}$ and $N_{v}^{i}$ in equation (2.5) and the choice of $L_{c}$ and $N_{c}$ in equation (2.7). In the former case, i.e. the univariate GARCH-MIDAS models, $L_{v}^{i}$ determines the number of lags spanned in each MIDAS polynomial specifications for $\tau_{t}$. The other is how to compute RV, weighted by the MIDAS polynomials. As pointed out by Engle, Ghysels, and Sohn (2006), this amounts to model selection with a fixed parameter space, and therefore is achieved via profiling the likelihood function for various combinations of $L_{v}^{i}$ and $N_{v}^{i}$. To determine the long run component of conditional correlations, $\overline{\boldsymbol{R}}_{t}$ we proceed in exactly the same way, namely we select the number of lags $L_{c}$ for historical correlations and the time span over which to compute the historical correlations $N_{c}$ in equation (2.7). The similarity between the two procedures is not surprising, given the fact that DCC models build extensively on the ideas of GARCH and in both cases we have a MIDAS filter extracting a component which behaves like a time-varying intercept. We will provide an explicit discussion of the procedure in the empirical applications, given the similarity with Engle, Ghysels, and Sohn (2006).

The asymptotic properties of the two-step estimator are discussed in Engle and Sheppard (2001), Comte and Lieberman (2003), Ling and McAleer (2003) and McAleer, Chan, Hoti, and Lieberman (2006). These papers deal with fixed parameter DCC models. It is beyond the scope of the current paper to establish the asymptotic properties of the MLE estimator when the MIDAS stochastic intercept is present. Recent work by Dahlhaus and Subba Rao (2006) discussed general time-varying coefficient $\mathrm{ARCH}(\infty)$ processes and regularity conditions for (local) MLE estimation. The GARCH-MIDAS and DCC-MIDAS processes are to a certain degree special cases of their setup. Namely, Dahlhaus and Subba Rao (2006) allow all parameters to vary and assume a nonparametric setting to capture the time-varying coefficients. This leads them, like Feng (2007), to consider kernel-based estimators. Our setting is parametric, as the MIDAS filter is a parametric specification, and therefore presumably simpler. We leave the regularity conditions that guarantee standard asymptotic results for the two-step estimation of DCC-MIDAS as an open question for future research. However, we do cover in this paper the regularity conditions we need to impose on the MIDAS-filtered long run correlation component to obtain positive definite matrices. This is the topic of the next section. 


\section{Regularity Conditions}

In this section, we turn our attention to the long run component and the choice of weights $\omega_{r}^{i j}$, keeping the lag lengths $N_{c}^{i j}$ and span lengths of historical correlations $L_{c}^{i j}$ fixed across all pairs of series. Hence, we focus on the memory decay in the long run correlations.

\subsection{Long-Run dynamics}

The first case to consider is the one of a common decay parameter $\omega_{r}$ independent of the pair of returns series selected. The covariance matrices can be shown to be positive definite under a relatively mild set of assumptions. When considering equation (2.11) it is apparent that the matrix $Q_{t}$ is a weighted average of three matrices. The matrix $\overline{\boldsymbol{R}}_{t}$ is a positive semi-definite because it is a weighted average of correlation matrices. The matrix $\boldsymbol{\xi}_{t} \boldsymbol{\xi}_{t}^{\prime}$ is always positive semi-definite by construction. Therefore, if the matrix $Q_{0}$ is initialized to a positive semi-definite matrix, it follows that $Q_{t}$ must be positive semi-definite at each point in time.

The positive semi-definiteness of the covariance matrix can be guaranteed without putting any restriction on the structure of the conditional variance estimators for the individual return series. This means, for example, that it is possible to assume a different number of GARCH-MIDAS lags for each return.

The case of two or more weighting schemes is more involved and therefore becomes more interesting. Indeed it is not always the case that the matrix $\overline{\boldsymbol{R}}_{t}\left(\underline{\omega}_{r}\right)$ is positive semi-definite for any choice of MIDAS parameters and specific restrictions on the parameter space ought to be imposed. The goal of this section is to provide sufficient conditions under which the sequence of matrices $\left\{\boldsymbol{\Phi}_{l}\right\}_{i=l}^{L_{c}}$ defined in (2.11) is positive semi-definite. Since the sequence of matrices $\left\{\boldsymbol{C}_{t-i}\right\}_{i=1}^{L_{c}}$ is positive semi-definite by construction, we can invoke the Schur product theorem to state that any matrix $\overline{\boldsymbol{R}}_{t}\left(\underline{\omega}_{r}\right)=\sum_{l=1}^{L_{c}} \boldsymbol{\Phi}_{l}\left(\underline{\omega}_{r}\right) \odot \boldsymbol{C}_{t-l}$ is positive semi-definite as well. ${ }^{7}$

In this section we examine the case of block matrices, whose dynamics can be accounted for by three parameters: the first two for block of correlations and the third

\footnotetext{
${ }^{7}$ For a proof of the Schur product theorem see Horn and Johnson (1985), theorem 7.5.3.
} 
one for the off-diagonal correlations. The following three definitions set the stage for the kind of matrices that we deal with in this section.

Definition 1 (Diagonal MIDAS Block). Let $\Phi_{l}^{D}\left(N_{a}, \omega_{r}^{a}\right)$ be a symmetric, square matrix of size $N_{a}$ such that all elements on the off-diagonal are equal to $\varphi_{l}\left(\omega_{r}^{a}\right)$ and all elements on the main diagonal are ones.

Definition 2 (Off-Diagonal MIDAS Block). Let $\boldsymbol{\Phi}_{l}^{F}\left(N_{a}, N_{b}, \omega_{r}^{c}\right)$ be a matrix of size $N_{a}, N_{b}$ such that all elements are equal to $\varphi_{l}\left(\omega_{r}^{c}\right)$.

Definition 3 (Block MIDAS matrix). Let

$$
\mathbf{\Phi}_{l}\left(\omega_{r}^{a}, \omega_{r}^{b}, \omega_{r}^{c}, N_{a}, N_{b}\right)=\left(\begin{array}{cc}
\boldsymbol{\Phi}_{l}^{D}\left(N_{a}, \omega_{r}^{a}\right) & \boldsymbol{\Phi}_{l}^{F}\left(N_{a}, N_{b}, \omega_{r}^{c}\right) \\
\boldsymbol{\Phi}_{l}^{F}\left(N_{a}, N_{b}, \omega_{r}^{c}\right)^{\prime} & \boldsymbol{\Phi}_{l}^{D}\left(N_{b}, \omega_{r}^{b}\right)
\end{array}\right)
$$

be a block matrix with $\boldsymbol{\Phi}_{l}^{D}\left(N_{a}, \omega_{r}^{a}\right)$ and $\boldsymbol{\Phi}_{l}^{D}\left(N_{b}, \omega_{r}^{b}\right)$ defined as in 1 and $\boldsymbol{\Phi}_{l}^{F}\left(N_{a}, N_{b}, \omega_{r}^{c}\right)$ defined as in 2.

The following lemmas lead up to the main proposition of this section.

Lemma 1. The determinant of the matrix $\Phi_{l}$ in Definition 3 is equal to

$$
\operatorname{det}\left(\mathbf{\Phi}_{l}\right)=\operatorname{det}_{A} \cdot \operatorname{det}_{B C A C}
$$

where

$$
\begin{aligned}
\operatorname{det}_{A} & =\operatorname{det}\left(\boldsymbol{\Phi}_{l}^{D}\left(N_{a}, \omega_{r}^{a}\right)\right) \\
\operatorname{det}_{B C A C} & =\operatorname{det}\left(\boldsymbol{\Phi}_{l}^{D}\left(N_{b}, \omega_{r}^{b}\right)-\mathbf{\Phi}_{l}^{F}\left(N_{a}, N_{b}, \omega_{r}^{c}\right)^{\prime} \boldsymbol{\Phi}_{l}^{D}\left(N_{a}, \omega_{r}^{a}\right)^{-1} \mathbf{\Phi}_{l}^{F}\left(N_{a}, N_{b}, \omega_{r}^{c}\right)\right)
\end{aligned}
$$

Proof. See Appendix.

This lemma suggests that in order to ensure the positive definiteness of each weighting matrix we can focus separately on the conditions that make the determinant of the first block matrix positive and on those that make the determinant of the function of matrices defined in (4.3) positive. We shall start by focusing on the first diagonal matrix.

Lemma 2. If $\varphi_{l}\left(\omega_{r}^{a}\right) \leq 1$ then all leading principal minors of $\Phi_{l}^{D}\left(N_{a}, \omega_{r}^{a}\right)$ are nonnegative. 
Proof. See Appendix.

According to the previous lemma, the only condition that needs to be verified for the leading principal minors of $\Phi_{l}$ up to the determinant of the first block matrix to be positive is that $\varphi_{l}\left(\omega_{r}^{a}\right)$ is less than one. This condition is always satisfied when using MIDAS filters.

The next two lemmas deal with the determinant of the function of sub-matrices defined in (4.3).

Lemma 3. If $\varphi_{l}\left(\omega_{r}^{a}\right) \geq 0$ and $\varphi_{l}\left(\omega_{r}^{b}\right) \leq 1$, the scalar

$$
l=\left(1-\varphi_{l}\left(\omega_{r}^{b}\right)\right)^{N_{b}-1}\left(1+\left(N_{b}-1\right) \varphi_{l}\left(\omega_{r}^{b}\right)\right)-N_{a} N_{b}\left[\varphi_{l}\left(\omega_{r}^{c}\right)\right]^{2}
$$

is always smaller than det $_{B C A C}$ defined in (4.3).

Proof. See Appendix.

Lemma 4. If $\varphi_{l}\left(\omega_{r}^{b}\right) \leq 1$ the function

$$
l\left(N_{b}\right)=\left(1-\varphi_{l}\left(\omega_{r}^{b}\right)\right)^{N_{b}-1}\left(1+\left(N_{b}-1\right) \varphi_{l}\left(\omega_{r}^{b}\right)\right)-N_{a} N_{b}\left[\varphi_{l}\left(\omega_{r}^{c}\right)\right]^{2}
$$

is always non-increasing in $N_{b}$.

Proof. See Appendix.

We are now ready to state the first of the two propositions of this sub-section.

Proposition 1. Let $\varphi_{l}\left(\omega_{r}^{a}\right)<1, \varphi_{l}\left(\omega_{r}^{b}\right)<1$, and $\varphi_{l}\left(\omega_{r}^{c}\right)<1$ and

$$
\left(1-\varphi_{l}\left(\omega_{r}^{b}\right)\right)^{N_{b}-1}\left(1+\left(N_{b}-1\right) \varphi_{l}\left(\omega_{r}^{b}\right)\right)-N_{a} N_{b}\left[\varphi_{l}\left(\omega_{r}^{c}\right)\right]^{2}>0
$$

the matrix $\boldsymbol{\Phi}_{l}\left(\omega_{r}^{a}, \omega_{r}^{b}, \omega_{r}^{c}, N_{a}, N_{b}\right)$ is positive definite.

Proof. Follows directly from lemmas 1, 2, and 4.

The assumption that $\varphi_{l}\left(\omega_{r}^{a}\right)<1, \varphi_{l}\left(\omega_{r}^{b}\right)<1$, and $\varphi_{l}\left(\omega_{r}^{c}\right)<1$ is always verified when using MIDAS filters, since they are all positive and are forced to sum up to one. 
Therefore, it amounts to checking that equation (4.5) is satisfied $\forall i$ to ensure that the weighting matrices are positive definite. This can amount to checking a nonnegligible amount of conditions in the case of lengthy MIDAS polynomial. For example, in the empirical applications, we show that the likelihood is maximized for 144 lags. A more useful theorem can be stated that amounts to checking only the first one of the conditions above. Its proof is a direct consequence of the following lemma and of proposition 1.

Lemma 5. Let $\varphi_{l}\left(\omega_{r}^{b}\right)>\varphi_{i+k}\left(\omega_{r}^{b}\right)$ and $\varphi_{l}\left(\omega_{r}^{c}\right)>\varphi_{i+k}\left(\omega_{r}^{c}\right)$ for some positive scalar $k$. Then

$$
l\left(\varphi_{l}\left(\omega_{r}^{b}\right), \varphi_{l}\left(\omega_{r}^{c}\right)\right) \leq l\left(\varphi_{i+k}\left(\omega_{r}^{b}\right), \varphi_{i+k}\left(\omega_{r}^{c}\right)\right)
$$

where the function $l(\cdot, \cdot)$ is defined in (4.4).

Proof. See Appendix.

Proposition 2. Let $\omega_{r}^{a}$, $\omega_{r}^{b}$, and $\omega_{r}^{c}$ be the characteristic parameters of the MIDAS filters $\left\{\varphi_{l}\left(\omega_{r}^{j}\right)\right\}_{i=1}^{I}, \forall j=\{a, b, c\}$ and let $\left\{\boldsymbol{\Phi}_{l}^{D}\left(N_{a}, \omega_{r}^{a}\right), \boldsymbol{\Phi}_{l}^{D}\left(N_{b}, \omega_{r}^{b}\right), \boldsymbol{\Phi}_{l}^{F}\left(N_{a}, N_{b}, \omega_{r}^{c}\right)\right\}_{i=1}^{I}$ be the associated sequences of block matrices according to definitions 1 and 2. If

$$
\left(1-\varphi_{1}\left(\omega_{r}^{b}\right)\right)^{N_{b}-1}\left[1+\left(N_{b}-1\right) \varphi_{1}\left(\omega_{r}^{b}\right)\right]-N_{a} N_{b}\left[\varphi_{1}\left(\omega_{r}^{c}\right)\right]^{2}>0
$$

then each matrix in the sequence $\left\{\boldsymbol{\Phi}_{l}\left(\omega_{r}^{a}, \omega_{r}^{b}, \omega_{r}^{c}, N_{a}, N_{b}\right)\right\}_{i=1}^{I}$ is positive definite.

Proof. Follows directly from proposition 1 , from lemma 5 and from the fact that each element of a MIDAS filter is bounded above by one.

This proposition conveniently states that in order to ensure the positive-definiteness of the long-run correlation matrix, one only needs to check one simple condition involving the first terms of the MIDAS polynomial. This condition is quite easily satisfied. For example, assume to have two sets of 10 series each (i.e. $N_{a}=N_{b}=10$ ): one that is better described by a long memory dynamics (say that $\omega_{r}^{a}=2$ ) and one that can be characterized as a short memory process (say that $\omega_{r}^{b}=15$ ). Also assume that the cross-correlations can be described as a MIDAS-average of the two process (i.e. say that $\omega_{r}^{c}=\frac{2+15}{2}$ ). The length of the MIDAS polynomial is $K_{c}=144$, a parameter that is shown to be optimal in the empirical application. Then, the left hand side 
of the condition of proposition 2 is equal to 0.4047 . This simple example documents that even in a 20 by 20 system, this parameterizations is quite flexible to ensure the positive definiteness of the resulting long-run correlation matrix.

The multiple-MIDAS filters cases that we analyze in the empirical section are all based on weighting matrices of the type:

$$
\Phi_{l}\left(\omega_{r}^{a}, \omega_{r}^{b}, \omega_{r}^{c}, N_{a}, N_{b}\right)=\left[\begin{array}{ccc}
1 & \varphi_{l}\left(\omega_{r}^{a}\right) & \varphi_{l}\left(\omega_{r}^{c}\right) \\
\varphi_{l}\left(\omega_{r}^{a}\right) & 1 & \varphi_{l}\left(\omega_{r}^{c}\right) \\
\varphi_{l}\left(\omega_{r}^{c}\right) & \varphi_{l}\left(\omega_{r}^{c}\right) & 1
\end{array}\right]
$$

where $N_{a}=2, N_{b}=1$, and $K_{c}=144$. The condition of proposition 2 boils down to $1-2 \varphi_{1}\left(\omega_{r}^{c}\right)^{2}>0$, which is always satisfied for $\omega_{r}^{c}<175$. Since a decay factor larger than 20 or 30 is hardly ever reached, we can state that this kind of 3 by 3 systems is always positive definite.

\subsection{Short-Run dynamics}

In general it will also prove convenient to allow for multiple sets of parameters to describe the DCC part of the correlation dynamics. In this subsection we study the positive semi-definiteness of the DCC part of the system, by imposing restrictions on the matrices of parameters $G, A$, and $B$ :

$$
\boldsymbol{Q}_{t}=G \odot \overline{\boldsymbol{R}}_{t}\left(\underline{\omega}_{r}\right)+A \odot \boldsymbol{\xi}_{t-1} \boldsymbol{\xi}_{t-1}^{\prime}+B \odot \boldsymbol{Q}_{t-1}
$$

We start with the case of three blocks of matrices.

Definition 4 (DCC Block matrices). Let $\left\{a_{i}\right\}_{i=1}^{3}$, and $\left\{b_{i}\right\}_{i=1}^{3}$ be positive scalars. The DCC block matrices, $A, B$ and $G$ are

$$
\begin{aligned}
& A=\left[\begin{array}{ll}
a_{1} \cdot \iota_{N_{j}}^{\prime} \iota_{N_{j}} & a_{3} \cdot \iota_{N_{j}}^{\prime} \iota_{N_{k}} \\
a_{3} \cdot \iota_{N_{k}}^{\prime} \iota_{N_{j}} & a_{2} \cdot \iota_{N_{k}}^{\prime} \iota_{N_{k}}
\end{array}\right], B=\left[\begin{array}{cc}
b_{1} \cdot \iota_{N_{j}}^{\prime} \iota_{N_{j}} & b_{3} \cdot \iota_{N_{j}}^{\prime} \iota_{N_{k}} \\
b_{3} \cdot \iota_{N_{k}}^{\prime} \iota_{N_{j}} & b_{2} \cdot \iota_{N_{k}}^{\prime} \iota_{N_{k}}
\end{array}\right], G=\iota_{N_{j}+N_{k}}^{\prime} \iota_{N_{j}+N_{k}}-A-B \\
& \text { where } \iota_{N_{l}}=[\underbrace{\left.\begin{array}{lll}
1 & \ldots & 1
\end{array}\right]}_{N_{l}}, \forall l \in\{j, k\} \text {. }
\end{aligned}
$$


The following three assumptions are needed in order to prove the positive semidefiniteness of the correlations matrices arising from the system above.

Assumption 1. Let $\left\{a_{i}\right\}_{i=1}^{3}$, and $\left\{b_{i}\right\}_{i=1}^{3}$ be non-negative scalars and let $1-a_{i}-b_{i} \geq 0$, $\forall i=\{1,2,3\}$.

Assumption 2. Let $a_{1} a_{2}-a_{3}^{2} \geq 0$ and $b_{1} b_{2}-b_{3}^{2} \geq 0$.

Assumption 3. The matrices $G \odot \overline{\boldsymbol{R}}_{t}\left(\underline{\omega}_{r}\right)$ are positive semi-definite.

Note that the first assumption is equivalent to the one needed to prove the existence of well-defined correlation matrices in the standard DCC model.

We are now ready to state the main theorem of this sub-section.

Proposition 3. Let the conditions of assumptions 1-3 be satisfied. Then the DCC block matrices, $A, B$, and $G$ are positive semi-definite.

Proof. Any principal minor that is the determinant of a sub-matrix of order equal or larger than three is zero, since it has at least two identical columns or rows. Any principal minor that is the determinant of a sub-matrix of order equal or larger than two is non-negative, because of assumptions 2 and 3 . The fact that all entries of the matrices are non-negative (by assumption 1) concludes the proof.

A special case of this specification is the Generalized-DCC model of Cappiello, Engle, and Sheppard (2003).

Definition 5 (Generalized DCC matrices). Let there be 2 pairs of DCC parameters $\left\{a_{j}, b_{j}\right\}_{j=1}^{2}$. The generalized DCC matrices, $A^{g}, B^{g}$, and $G^{g}$ are

$$
A^{g}=\boldsymbol{a} \boldsymbol{a}^{\prime}, B^{g}=\boldsymbol{b} \boldsymbol{b}^{\prime}, G^{g}=\left(\boldsymbol{\iota} \boldsymbol{\iota}^{\prime}-\boldsymbol{a} \boldsymbol{a}^{\prime}-\boldsymbol{b} \boldsymbol{b}^{\prime}\right)
$$

where

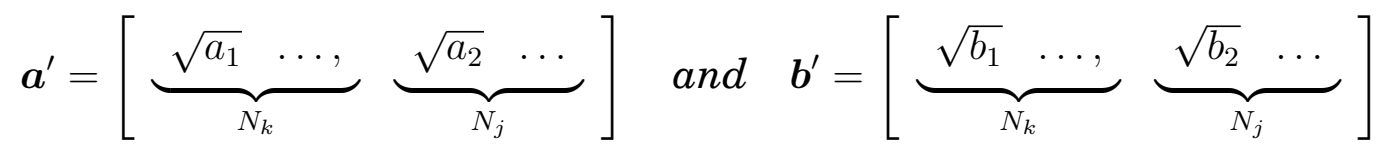


This specification satisfies assumption 2 above with equality, but there is no specific reason why that should be the case and, in any event, this is a testable restriction. Therefore the DCC block structure provides a more flexible specification, that under mild assumptions delivers well-defined correlation matrices.

\section{Empirical Applications}

We study various empirical examples, starting with a bi-variate system, next moving to two examples with three assets. For the equities, the portfolios are formed based on an industry classification ${ }^{8}$ In the bi-variate case, we only have one MIDAS filter, hence the discussion pertaining to regularity conditions for positive semi-definiteness in the previous section does not apply. We therefore move to the tri-variate systems to discuss the selection of MIDAS filters. Each of the examples are designed to highlight model estimation and specification issues. The section is divided in subsections which cover the various examples.

\subsection{An example of two assets: Energy portfolio and 10 year bond}

We start the investigation of short and long run correlation dynamics of industries portfolios and 10 year bond, by focusing on a bi-variate case that involves the energy industry portfolio and the 10 year bond only. The energy industry portfolio collects stocks related to oil, gas, and coal extraction and products. It is convenient to outline the various steps involved in the estimation procedure in the context of this simple case.

We consider a sample of daily returns on an industry portfolio - the energy sector and a long term bond, namely a 10-year bond. The sample starts on 1971-07-15 and ends on 2006-06-30. We first address the issue of selecting the number of MIDAS

\footnotetext{
${ }^{8}$ Data were downloaded from Kenneth French web site and correspond to the 10 industry portfolio classification. Accordingly, each NYSE, AMEX, and NASDAQ stock is assigned to an industry portfolio based on its four-digit SIC code. We report the details on the specific portfolios that we employed in our empirical analysis in the Appendix.
} 
lags. We follow a procedure suggested in Engle, Ghysels, and Sohn (2006), since the GARCH-MIDAS and DCC-MIDAS class of models share similar model selection issues. A convenient property of both models, is that the lag selection of the MIDAS filter involves a fixed number of parameters. Hence, Engle, Ghysels, and Sohn (2006) compare various GARCH-MIDAS models with different time spans via profiling of the likelihood function. The task can be easily accomplished by looking at the plots of the log-likelihood functions of the GARCH-MIDAS and DCC-MIDAS estimators for an increasing number of lags. The left panel of Figure 1 shows that a small number of MIDAS lags is typically enough to accurately describe the long run dynamics of the volatilities of the two series. We select the smallest number of MIDAS lags after which the log-likelihoods of the two volatilities seem to reach their plateau. In this case this criterion amounts to picking 36 lags of monthly realized volatilities. The right panel of Figure 1 shows that DCC-MIDAS requires a larger number of lags before its log-likelihood flattens out. This led us to select 144 lags.

Figure 2 reports the short and long run dynamics of volatilities and correlations. The dark lines in the figure correspond to the long run correlation with short run correlations snaking around it. These results show that despite the fact that the unconditional correlation of the two series is close to zero, the short-run correlation is characterized by large departures from this value. The long-run correlation slowly adjusts to account for periods of relatively higher and relatively lower correlation. Table 2 reports the estimates of the parameters of the DCC-MIDAS and of the original DCC, where the latter is modified to take into account the long-run dynamics of volatilities only. It is interesting to note that the persistence parameters $\beta$ of the two GARCH processes are remarkably lower than we typically observe when neglecting the long-run dynamics for daily series. The same is true also for the persistence parameter of the correlation $b$. Both facts can be attributed to the slowly moving MIDAS adjustment.

Figure 3 shows how much the short run correlations are affected by ignoring timevarying long-run dynamics. The solid line represents the differences in correlations between the original DCC and the DCC-MIDAS. These differences can be as high as 0.05 in absolute value and the picture shows that they are typically positive when the time varying long run correlation exceeds the unconditional one and negative when the relationship between the two correlations is reversed. 


\subsection{More than two assets}

When we consider more than two assets we have the possibility that several long run MIDAS filters as well as multiple DCC parameters apply. ${ }^{9}$ We provide two examples involving three asset returns, one where a single MIDAS filter suffices, and another where there is clearly a need for two filters. The former involves two industries and a bond, namely Energy and Hi-Tech portfolios vs. 10 year bond. The results appear in Figures 4 and 5 as well as Table 3. The first figure displays Energy-HiTech-10 year Bond Variances and correlation and the second shows the differences with DCC and unconditional correlations.

In Table 5 we report likelihood ratio tests for various nested model specifications involving separate parameters for the DCC dynamics and/or MIDAS filters. Each entry in the table represents the p-value for testing that the likelihood of the model of the column is significantly higher than the likelihood of the model on the corresponding row. The first row of the table documents that the baseline one DCC - one MIDAS model may not be enough to account for the dynamics of the system. The specifications with two sets of DCC parameters seem to yield significant lower likelihoods in all pairwise comparisons. It is also the case that adding an additional MIDAS parameter does not improve the model performance when a second pair of DCC parameters has already been added. This is always true with the only possible exception of the comparison between one and two MIDAS with two sets of non-generalized DCC parameters. The model with three distinct sets of DCC parameters does not appear to significantly improve the likelihood.

These results convey that one MIDAS parameter is enough to account for the longrun dynamics of the system, confirming the idea that one might have had by looking at the small absolute differences in the estimated bi-variate models. The short-run dynamics is the one needing a more flexible specification in this example. A model with two sets of DCC parameters and one MIDAS parameter seems to suffice to accurately describe the joint dynamics of the three assets. However we cannot draw any conclusion as to whether we should employ the generalized or non-generalized DCC structure on the grounds of the likelihood ratio tests, being the two models non

\footnotetext{
${ }^{9}$ The appendix reports the specifics and the names of the models that are being estimated in this subsection.
} 
nested. The two left-most columns of table 4 show that the generalized specification with two sets of DCC parameters and one MIDAS appears to perform better than its non-generalized counterpart, according to the AIC and BIC criteria.

The next and final example shows that this is not always the case. In Table 8 we report likelihood ratio tests for Ten year Bonds combined with Manufacturing and Shops industries. The parameter estimates, for the single MIDAS filter appear in Table 6 whereas the multiple filter case appears in Table 7. The bottom part of the table shows that when the MIDAS parameters are estimated in all possible permutations of bivariate systems, a value close to 7 appears to do the job for bond vs. manufacturing and for for bond vs. shops. A decisively shorter memory achieves the maximum likelihood when it comes to accounting for the long run dynamics of the correlation between shops and manufacturing. The top part of table 7 shows that it can indeed be quite restrictive to force one MIDAS parameter to describe the long-run dynamics of all pairs of correlations. The introduction of an additional MIDAS parameter not only brings the outcome of the estimation closer to what suggested by the analysis of the bivariate systems, but it also sizeably increases the log-likelihood. Table 8 shows that this increase is significant at a $1 \%$ confidence level.

The variances and correlations appear respectively in Figures 6 and 8. The former shows the single filter patterns and the latter shows the patterns with two distinct filters. We observe that the second filter clearly changes the long run component correlation across the two industries. Figure 5 as well as figure 7 confirm once again that the original DCC seems to overshoot on average the short run correlation when the long run one is above its unconditional value, while the opposite happens when the MIDAS long run correlation lies below its unconditional counterpart.

When we employ the Generalized DCC-MIDAS model, we obtain the parameters' estimates reported in table 7 . These estimates seem to confirm the need for a second MIDAS filter to be applied to the correlation between the manufacturing and the shops portfolios. Figure 9 shows that the long-run correlations filtered using this specification appear to be a little smoother when the 10-year bond is one of the assets compared to the results obtained under the previous specification. The low-frequency correlation of the two portfolios is instead a little noisier. Aside for these small differences, we take the results as confirming the need for multiple set of correlation parameters. 


\section{Concluding remarks}

We introduced a class of DCC-MIDAS component models of dynamic correlations with a short- and long-run component specification. The key ingredients are a combination of the Engle (2002) DCC model, the Engle and Lee (1999) component GARCH model to replace the original DCC dynamics with a component specification and the Engle, Ghysels, and Sohn (2006) GARCH-MIDAS component specification that allows us to extract a long-run correlation component via mixed data sampling. We addressed the specification, estimation and interpretation of correlation models that distinguish short and long run components. We show that the changes in correlations are indeed very different. An empirical illustration shows the benefits of the component specification. Empirical specification tests are introduced and applied. They reveal the superior empirical fit of the new class of DCC-MIDAS correlation models. While we left the regularity conditions that guarantee standard asymptotic results for the twostep estimation of DCC-MIDAS as an open question for future research we did cover one important part of the regularity conditions dealing with the positive definiteness of the MIDAS-filtered long run correlation component.

\section{References}

Comte, F. and O. Lieberman (2003). Asymptotic theory for multivariate garch processes. Journal of Multivariate Analysis 84, 61-84.

Dahlhaus, R. and S. Subba Rao (2006). Statistical inference for time-varying arch processes. Annals of Statistics 34, 1075-1114.

Engle, R. (2002). Dynamic conditional correlation - a simple class of multivariate garch models. Journal of Business and Economic Statistics.

Engle, R., E. Ghysels, and B. Sohn (2006). On the economic sources of stock market volatility. NYU and UNC unpublished manuscript. 
Engle, R. and G. Lee (1999). A permanent and transitory component model of stock return volatility. in ed. R.F. Engle and H. White, Cointegration, Causality, and Forecasting: A Festschrift in Honor of Clive W.J. Granger, (Oxford University Press), 475-497.

Engle, R. and J. Rangel (2005). The spline garch model for unconditional volatility and its global macroeconomic causes. manuscript NYU and UCSD.

Engle, R. and K. Sheppard (2001). Theoretical and empirical properties of dynamic conditional correlation multivariate garch. Discussion Paper, UCSD.

Feng, Y. (2007). A local dynamic conditional correlation model. MPRA Paper No. 1592.

Forsberg, L. and E. Ghysels (2004). Why do absolute returns predict volatility so well? Journal of Financial Econometrics, forthcoming.

Ghysels, E., P. Santa-Clara, and R. Valkanov (2005). There is a risk-return tradeoff after all. Journal of Financial Economics 76, 509-548.

Ghysels, E., P. Santa-Clara, and R. Valkanov (2006). Predicting volatility: getting the most out of return data sampled at different frequencies. Journal of Econometrics 131, 59-95.

Horn, R. A. and C. R. Johnson (1985). Matrix analysis. Cambridge University Press.

Karolyi, G. and R. Stulz (1996). Why do markets move together? an investigation of u.s.-japan stock return comovements. Journal of Finance 51, 951-986.

Ling, S. and M. McAleer (2003). Asymptotic theory for a new vector arma-garch model. Econometric Theory 19, 280-310.

McAleer, M., F. Chan, S. Hoti, and O. Lieberman (2006). Generalized autoregressive conditional correlation. Pre-print.

Schwert, G. W. (1989). Why does stock market volatility change over time? Journal of Finance 44, 1207-1239. 


\section{Technical Appendix}

\section{Proofs of Lemmas}

Proof of Lemma 1. Follows directly from decomposing the matrix as

$$
\begin{aligned}
\boldsymbol{\Phi}_{l}= & \left(\begin{array}{cc}
\boldsymbol{\Phi}_{l}^{D}\left(N_{a}, \omega_{r}^{a}\right) & \mathbf{0} \\
\boldsymbol{\Phi}_{l}^{F}\left(N_{a}, N_{b}, \omega_{r}^{c}\right)^{\prime} & \boldsymbol{I}_{N_{b}}
\end{array}\right) . \\
& \left(\begin{array}{cc}
\boldsymbol{I}_{N_{a}} & \boldsymbol{\Phi}_{l}^{D}\left(N_{a}, \omega_{r}^{a}\right)^{-1} \boldsymbol{\Phi}_{l}^{F}\left(N_{a}, N_{b}, \omega_{r}^{c}\right) \\
\mathbf{0} & \boldsymbol{\Phi}_{l}^{D}\left(N_{b}, \omega_{r}^{b}\right)-\boldsymbol{\Phi}_{l}^{F}\left(N_{a}, N_{b}, \omega_{r}^{c}\right)^{\prime} \boldsymbol{\Phi}_{l}^{D}\left(N_{a}, \omega_{r}^{a}\right)^{-1} \boldsymbol{\Phi}_{l}^{F}\left(N_{a}, N_{b}, \omega_{r}^{c}\right)
\end{array}\right)
\end{aligned}
$$

Proof of Lemma 2. It follows directly from observing that any leading principal minor of $\boldsymbol{\Phi}_{l}^{D}\left(N_{a}, \omega_{r}^{a}\right)$ can be written as

$$
\operatorname{det}_{k}=\left(1-\varphi_{l}\left(\omega_{r}^{a}\right)\right)^{k-1}\left(1+(k-1) \varphi_{l}\left(\omega_{r}^{a}\right)\right), \quad \forall k \geq 1
$$

Proof of Lemma 3. Since

$$
\operatorname{det}_{B C A C}=-N_{a} N_{b} \frac{\left(1-\varphi_{l}\left(\omega_{r}^{b}\right)\right)^{N_{b}-1}}{1+\left(1+N_{a}\right) \varphi_{l}\left(\omega_{r}^{a}\right)}\left[\varphi_{l}\left(\omega_{r}^{c}\right)\right]^{2}+\left(1-\varphi_{l}\left(\omega_{r}^{b}\right)\right)^{N_{b}-1}\left(1+\left(N_{b}-1\right) \varphi_{l}\left(\omega_{r}^{b}\right)\right)
$$

it amounts to showing that

$$
\frac{\left(1-\varphi_{l}\left(\omega_{r}^{b}\right)\right)^{N_{b}-1}}{1+\left(1+N_{a}\right) \varphi_{l}\left(\omega_{r}^{a}\right)} \leq 1
$$

This is always the case since the numerator is always smaller than unity (because $\varphi_{l}\left(\omega_{r}^{b}\right) \leq$ 1 by assumption) and the denominator is always larger than one (because $\varphi_{l}\left(\omega_{r}^{a}\right) \geq 0$ by assumption).

Proof of Lemma 4. Denote $l\left(N_{b}\right)$ as the difference

$$
l\left(N_{b}\right)=p\left(N_{b}\right)-q\left(N_{b}\right)
$$


where

$$
\begin{aligned}
& p\left(N_{b}\right)=\left(1-\varphi_{l}\left(\omega_{r}^{b}\right)\right)^{N_{b}-1}\left(1+\left(N_{b}-1\right) \varphi_{l}\left(\omega_{r}^{b}\right)\right) \\
& q\left(N_{b}\right)=N_{a} N_{b}\left[\varphi_{l}\left(\omega_{r}^{c}\right)\right]^{2}
\end{aligned}
$$

The term $-q\left(N_{b}\right)$ is trivially always decreasing in $N_{b}$. The term $p\left(N_{b}\right)$ can be written as

$$
p\left(N_{b}\right)=1+\left[\varphi_{l}\left(\omega_{r}^{b}\right)\right]^{2} \sum_{j=1}^{N_{b}-1}(-1)^{j} \cdot j \cdot\left(\varphi_{l}\left(\omega_{r}^{b}\right)-1\right)^{j-1}
$$

with $p\left(N_{b}=1\right)=1$. The increments:

$$
p\left(N_{b}\right)-p\left(N_{b}-1\right)=\left[\varphi_{l}\left(\omega_{r}^{b}\right)\right]^{2}(-1)^{N_{b}-1} \cdot\left(N_{b}-1\right) \cdot\left(\varphi_{l}\left(\omega_{r}^{b}\right)-1\right)^{N_{b}-2}
$$

are always negative, because if $N_{b}$ is odd (even), the term $(-1)^{N_{b}-1}$ is positive (negative), while the term $\left(\varphi_{l}\left(\omega_{r}^{b}\right)-1\right)^{N_{b}-2}$ is negative (positive), since $\varphi_{l}\left(\omega_{r}^{b}\right) \leq 1$, by assumption.

Proof of Lemma 5. Decompose $l\left(\varphi_{l}\left(\omega_{r}^{b}\right), \varphi_{l}\left(\omega_{r}^{c}\right)\right)$ as

$$
l\left(\varphi_{l}\left(\omega_{r}^{b}\right), \varphi_{l}\left(\omega_{r}^{c}\right)\right)=p\left(\varphi_{l}\left(\omega_{r}^{b}\right)\right)-q\left(\varphi_{l}\left(\omega_{r}^{c}\right)\right)
$$

where

$$
\begin{aligned}
p\left(\varphi_{l}\left(\omega_{r}^{b}\right)\right) & =1+\sum_{j=1}^{N_{b}-1}\left[\varphi_{l}\left(\omega_{r}^{b}\right)\right]^{2}(-1)^{j} \cdot j \cdot\left[\varphi_{l}\left(\omega_{r}^{b}\right)\right]^{j-1} \\
& =1+\sum_{j=1}^{N_{b}-1} p_{j}\left(\varphi_{l}\left(\omega_{r}^{b}\right)\right) \\
q\left(\varphi_{l}\left(\omega_{r}^{c}\right)\right) & =N_{a} N_{b}\left[\varphi_{l}\left(\omega_{r}^{c}\right)\right]^{2}
\end{aligned}
$$

The term $-q\left(\varphi_{l}\left(\omega_{r}^{c}\right)\right)$ is trivially decreasing in $\varphi_{l}\left(\omega_{r}^{c}\right)$. For the other term:

$$
\begin{aligned}
p_{j}\left(\varphi_{l}\left(\omega_{r}^{b}\right)\right) & =-\left[\varphi_{l}\left(\omega_{r}^{b}\right)\right]^{2}(-1)^{j} \cdot j \cdot\left|\varphi_{l}\left(\omega_{r}^{b}\right)\right|^{j-1} \\
& \leq-\left[\varphi_{i+k}\left(\omega_{r}^{b}\right)\right]^{2}(-1)^{j} \cdot j \cdot\left|\varphi_{i+k}\left(\omega_{r}^{b}\right)\right|^{j-1}=p_{j}\left(\varphi_{i+k}\left(\omega_{r}^{b}\right)\right), \quad \forall j=\left\{1, \cdots, N_{b}-1\right\}
\end{aligned}
$$




\section{Details on industry portfolios classification}

Data are download from Kenneth French web-site. The Energy, Manufacturing, Hi-Tech, and Shops portfolios that we use in the empirical section correspond to the collection of the following SIC codes:

1. Energy: Oil, Gas, and Coal Extraction and Products SIC codes: 1200-1399, 2900-2999

2. Manufacturing: Machinery, Trucks, Planes, Chemicals, Off Furn, Paper, Com Printing SIC codes: 2520-2589, 2600-2699, 2750-2769, 2800-2829, 2840-2899, 3000-3099, 32003569, 3580-3621, 3623-3629, 3700-3709, 3712-3713, 3715-3715, 3717-3749, 3752-3791, 3793-3799, 3860-3899

3. Hi-Tech: Computers, Software, and Electronic Equipment, Industrial controls, computer programming and data processing, Computer integrated systems design, computer processing, data prep, information retrieval services, computer facilities management service, computer rental and leasing, computer maintenance and repair, computer related services, $R \& D$ labs, research, development, testing labs SIC codes: 3570-3579, 3622-3622, 3660-3692, 3694-3699, 3810-3839, 7370-7372, 73737373, 7374-7374, 7375-7375, 7376-7376, 7377-7377, 7378-7378, 7379-7379, 7391-7391, $8730-8734$

4. Shops: Wholesale, Retail, and Some Services (Laundries, Repair Shops) SIC codes: 5000-5999, 7200-7299, 7600-7699

\section{Summary of specifications}

In the empirical section, we will analyze the performance of several combinations of short- and long-run specifications for a number of 3 by 3 systems. To simplify the reading of the results, we summarize and label the models in this sub-section.

1. MIDAS=1: the typical MIDAS correlation weighting matrix is

$$
\boldsymbol{\Phi}_{l}\left(\omega_{1}\right)=\left[\begin{array}{ccc}
1 & \varphi_{l}\left(\omega_{1}\right) & \varphi_{l}\left(\omega_{1}\right) \\
\varphi_{l}\left(\omega_{1}\right) & 1 & \varphi_{l}\left(\omega_{1}\right) \\
\varphi_{l}\left(\omega_{1}\right) & \varphi_{l}\left(\omega_{1}\right) & 1
\end{array}\right]
$$


2. MIDAS=2: the typical MIDAS correlation weighting matrix is

$$
\Phi_{l}\left(\omega_{1}, \omega_{2}\right)=\left[\begin{array}{ccc}
1 & \varphi_{l}\left(\omega_{2}\right) & \varphi_{l}\left(\omega_{1}\right) \\
\varphi_{l}\left(\omega_{2}\right) & 1 & \varphi_{l}\left(\omega_{1}\right) \\
\varphi_{l}\left(\omega_{1}\right) & \varphi_{l}\left(\omega_{1}\right) & 1
\end{array}\right]
$$

Hence, the second MIDAS polynomial insists on the correlation of the first two assets with the third asset.

3. $\mathrm{DCC}=1$ : the short-run dynamics are governed by the scalars $a_{1}$ and $b_{1}$.

4. $\mathrm{DCC}=2$ : the short-run dynamics are described by the following matrices:

$$
A=\left[\begin{array}{lll}
a_{2} & a_{2} & a_{1} \\
a_{2} & a_{2} & a_{1} \\
a_{1} & a_{1} & a_{1}
\end{array}\right] \quad B=\left[\begin{array}{lll}
b_{2} & b_{2} & b_{1} \\
b_{2} & b_{2} & b_{1} \\
b_{1} & b_{1} & b_{1}
\end{array}\right]
$$

5. $\mathrm{DCC}=3$ : the short-run dynamics are described by the following matrices:

$$
A=\left[\begin{array}{lll}
a_{2} & a_{2} & a_{3} \\
a_{2} & a_{2} & a_{3} \\
a_{3} & a_{3} & a_{1}
\end{array}\right] \quad B=\left[\begin{array}{lll}
b_{2} & b_{2} & b_{3} \\
b_{2} & b_{2} & b_{3} \\
b_{3} & b_{3} & b_{1}
\end{array}\right]
$$

6. $\mathrm{DCC}=2(\mathrm{G})$ : the short-run dynamics are described by the following matrices:

$$
A=\left[\begin{array}{ccc}
a_{2} & a_{2} & \sqrt{a_{1} a_{2}} \\
a_{2} & a_{2} & \sqrt{a_{1} a_{2}} \\
\sqrt{a_{1} a_{2}} & \sqrt{a_{1} a_{2}} & a_{1}
\end{array}\right] \quad B=\left[\begin{array}{ccc}
b_{1} & b_{1} & \sqrt{b_{1} b_{2}} \\
b_{1} & b_{1} & \sqrt{b_{1} b_{2}} \\
\sqrt{b_{1} b_{2}} & \sqrt{b_{1} b_{2}} & b_{2}
\end{array}\right]
$$


TABLE 1

MeAn Absolute ERrors

\begin{tabular}{lccccccc}
\hline \hline & Step & Large Step & Dbl Step & Dbl Step Long & Ramp & Short Cycle & Long Cycle \\
\hline DCC & 0.072 & 0.113 & 0.115 & 0.118 & 0.159 & 0.120 & 0.100 \\
Original & $(.056, .091)$ & $(.093, .142)$ & $(.101, .132)$ & $(.100, .136)$ & $(.142, .173)$ & $(.106, .134)$ & $(.084, .122)$ \\
\hline DCC & 0.070 & 0.107 & 0.114 & 0.116 & 0.160 & 0.120 & 0.101 \\
MIDAS & $(.053, .097)$ & $(.088, .133)$ & $(.099, .133)$ & $(.099, .133)$ & $(.143, .174)$ & $(.106, .134)$ & $(.084, .122)$ \\
\hline
\end{tabular}

Notes - Each entry represents the average Mean Absolute Error (MAE) obtained by simulating correlations using the pattern reported in the corresponding column and estimating them through the model in the corresponding row. The numbers in parenthesis are the $95 \%$ confidence intervals.

\section{TABLE 2}

\section{ENERGY PORTFOLIO VS. 10 YEAR BOND}

\begin{tabular}{lcccccc}
\hline \hline & $\mu$ & $\alpha$ & $\beta$ & $\theta$ & $\omega$ & $m$ \\
\hline Energy & 0.069 & 0.084 & 0.812 & 0.198 & 12.459 & 0.548 \\
& $(0.077)$ & $(0.021)$ & $(0.006)$ & $(0.027)$ & $(0.000)$ & $(0.049)$ \\
\hline Bond & 0.022 & 0.058 & 0.919 & 0.203 & 2.566 & 0.296 \\
& $(0.011)$ & $(0.000)$ & $(0.006)$ & $(0.018)$ & $(0.001)$ & $(0.075)$ \\
\hline
\end{tabular}

\begin{tabular}{lccc} 
& $a$ & $b$ & $\omega$ \\
\hline DCC-MIDAS & 0.015 & 0.979 & 1.774 \\
& $(0.001)$ & $(0.003)$ & $(0.469)$ \\
\hline DCC & 0.015 & 0.981 & - \\
& $(0.000)$ & $(0.000)$ & - \\
\hline
\end{tabular}

Notes - The top panel reports the estimates of the GARCH-MIDAS coefficients for the Energy portfolio and 10 year Bond. The bottom panel reports the estimates of the DCC-MIDAS and original DCC parameters. The number of MIDAS lags is 36 for the GARCH processes and 144 for the DCC process. The sample covers 1971-07-15 until 2006-06-30. 
TABLE 3

ENERGY, HI-TECH AND 10 YEAR BOND

\begin{tabular}{lcccccc}
\hline \hline & $\mu$ & $\alpha$ & $\beta$ & $\theta$ & $\omega$ & $m$ \\
\hline Energy & 0.070 & 0.087 & 0.804 & 0.199 & 12.602 & 0.545 \\
& $(0.000)$ & $(0.000)$ & $(0.016)$ & $(0.065)$ & $(0.000)$ & $(0.123)$ \\
\hline Hi-Tech & 0.063 & 0.087 & 0.837 & 0.186 & 9.997 & 0.726 \\
& $(0.000)$ & $(0.064)$ & $(0.001)$ & $(0.000)$ & $(0.000)$ & $(0.332)$ \\
\hline Bond & 0.022 & 0.059 & 0.915 & 0.204 & 3.090 & 0.284 \\
& $(0.002)$ & $(0.000)$ & $(0.000)$ & $(0.002)$ & $(0.000)$ & $(0.000)$ \\
\hline
\end{tabular}

\begin{tabular}{lccc} 
& $a$ & $b$ & $\omega$ \\
\hline DCC-MIDAS & 0.018 & 0.977 & 1.683 \\
& $(0.004)$ & $(0.000)$ & $(0.000)$ \\
\hline DCC & 0.016 & 0.981 & - \\
& $(0.001)$ & $(0.001)$ & - \\
\hline
\end{tabular}

Notes - The top panel reports the estimates of the GARCH-MIDAS coefficients for the Energy portfolio, Hi-Tech portfolio and 10 year Bond. The bottom panel reports the estimates of the DCC-MIDAS and original DCC parameters. The number of MIDAS lags is 36 for the GARCH processes and 144 for the DCC process. The sample covers 1971-07-15 until 2006-06-30. 


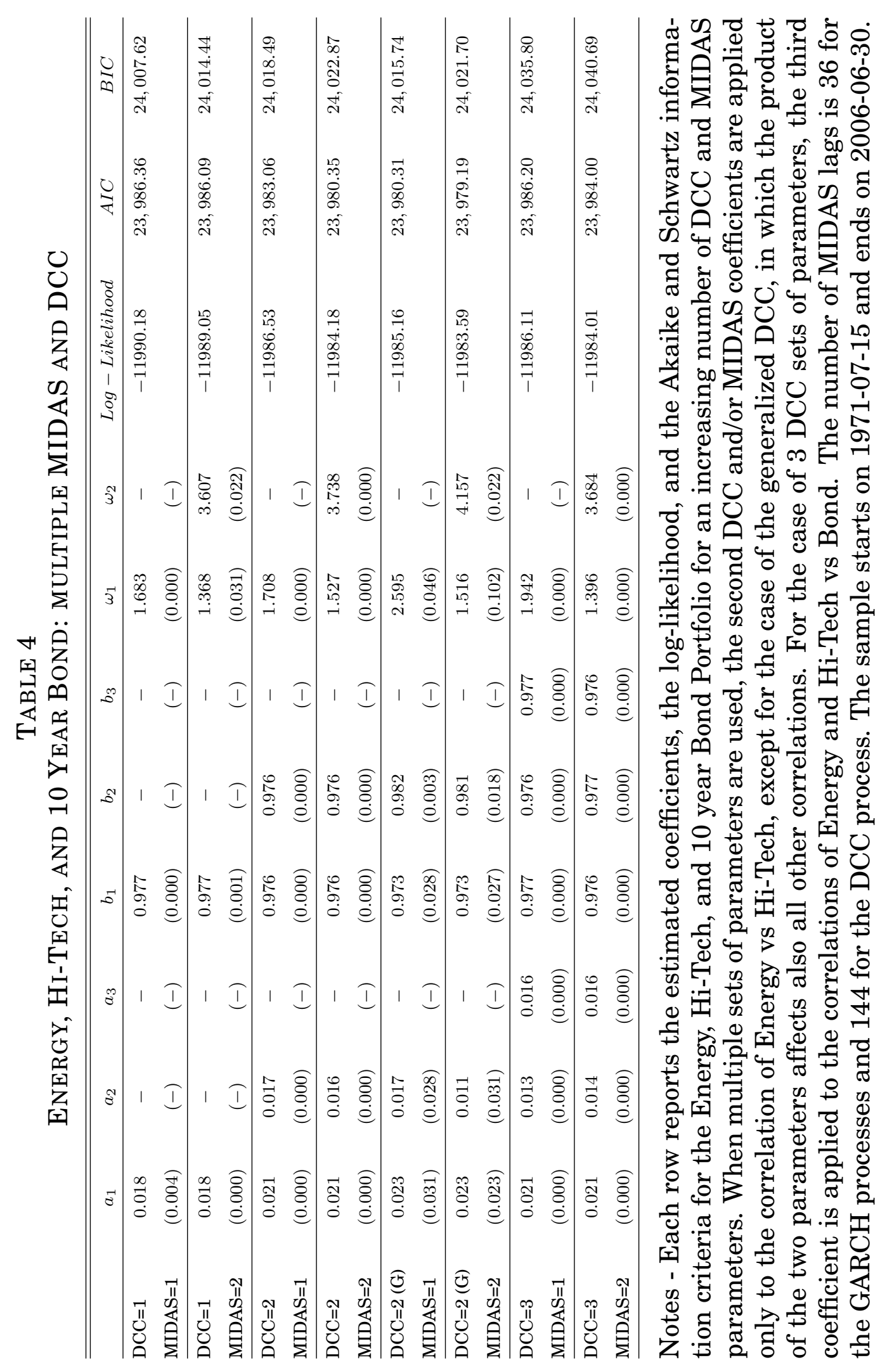




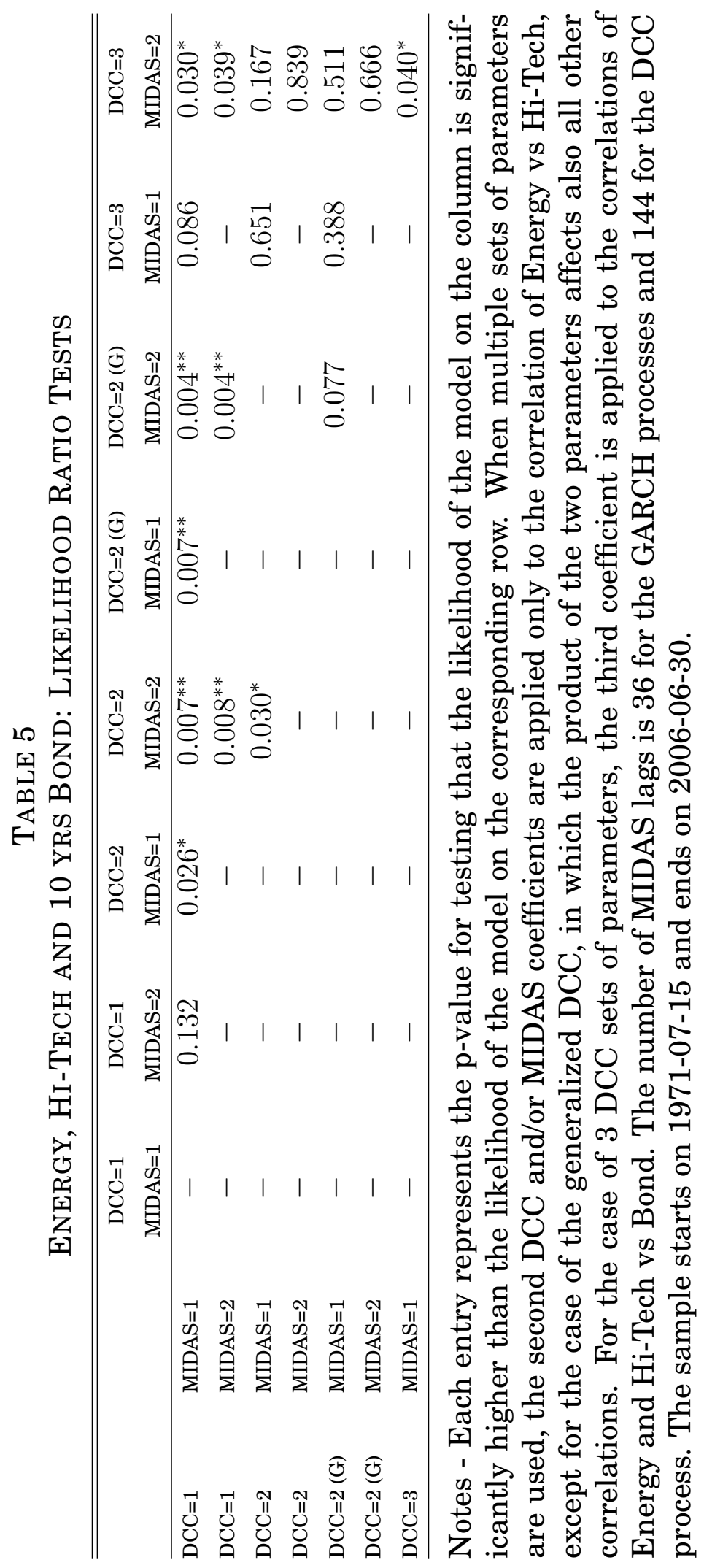


TABLE 6

MANUFACTURING, SHOPS, AND 10 YeAR BOND

\begin{tabular}{lcccccc}
\hline \hline & $\mu$ & $\alpha$ & $\beta$ & $\theta$ & $\omega$ & $m$ \\
\hline Bond & 0.022 & 0.059 & 0.914 & 0.204 & 3.090 & 0.284 \\
& $(0.001)$ & $(0.000)$ & $(0.000)$ & $(0.002)$ & $(0.000)$ & $(0.000)$ \\
\hline Manufacturing & 0.072 & 0.103 & 0.801 & 0.175 & 10.925 & 0.564 \\
& $(0.000)$ & $(0.000)$ & $(0.078)$ & $(0.019)$ & $(0.005)$ & $(0.082)$ \\
\hline Shops & 0.069 & 0.101 & 0.816 & 0.171 & 11.529 & 0.632 \\
& $(0.001)$ & $(0.002)$ & $(0.000)$ & $(0.003)$ & $(0.001)$ & $(0.013)$ \\
\hline
\end{tabular}

\begin{tabular}{lccc} 
& $a$ & $b$ & $\omega$ \\
\hline DCC-MIDAS & 0.029 & 0.954 & 11.680 \\
& $(0.000)$ & $(0.000)$ & $(1.215)$ \\
\hline DCC & 0.217 & 0.975 & - \\
& $(0.000)$ & $(0.000)$ & - \\
\hline
\end{tabular}

Notes - The top panel reports the estimates of the GARCH-MIDAS coefficients for the 10 year Bond, Manufacturing portfolio, and the Shops portfolio. The bottom panel reports the estimates of the DCC-MIDAS and of the original DCC parameters. The number of MIDAS lags is 36 for the GARCH processes and 144 for the DCC process. The sample covers 1971-07-15 until 2006-06-30. 


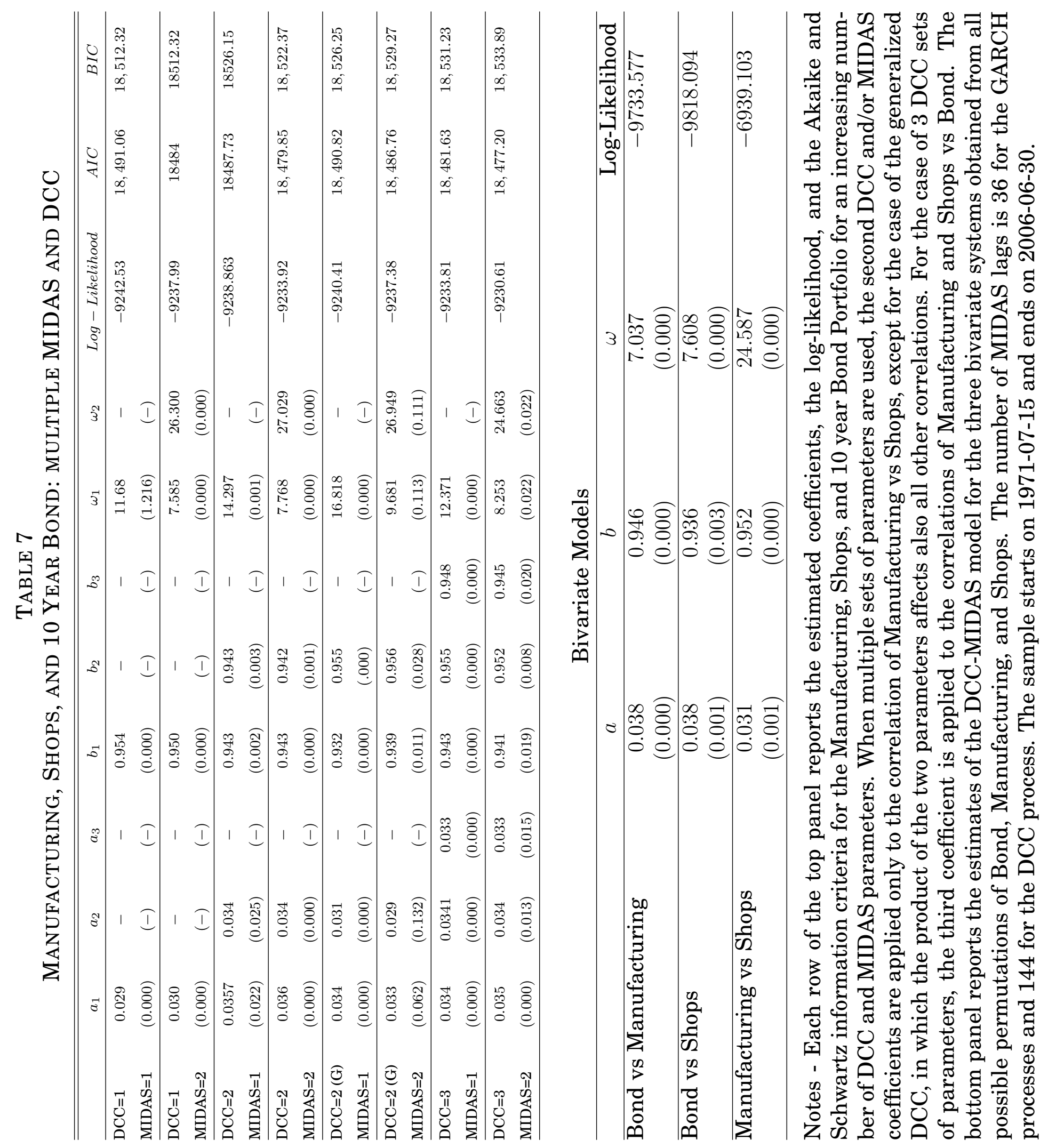




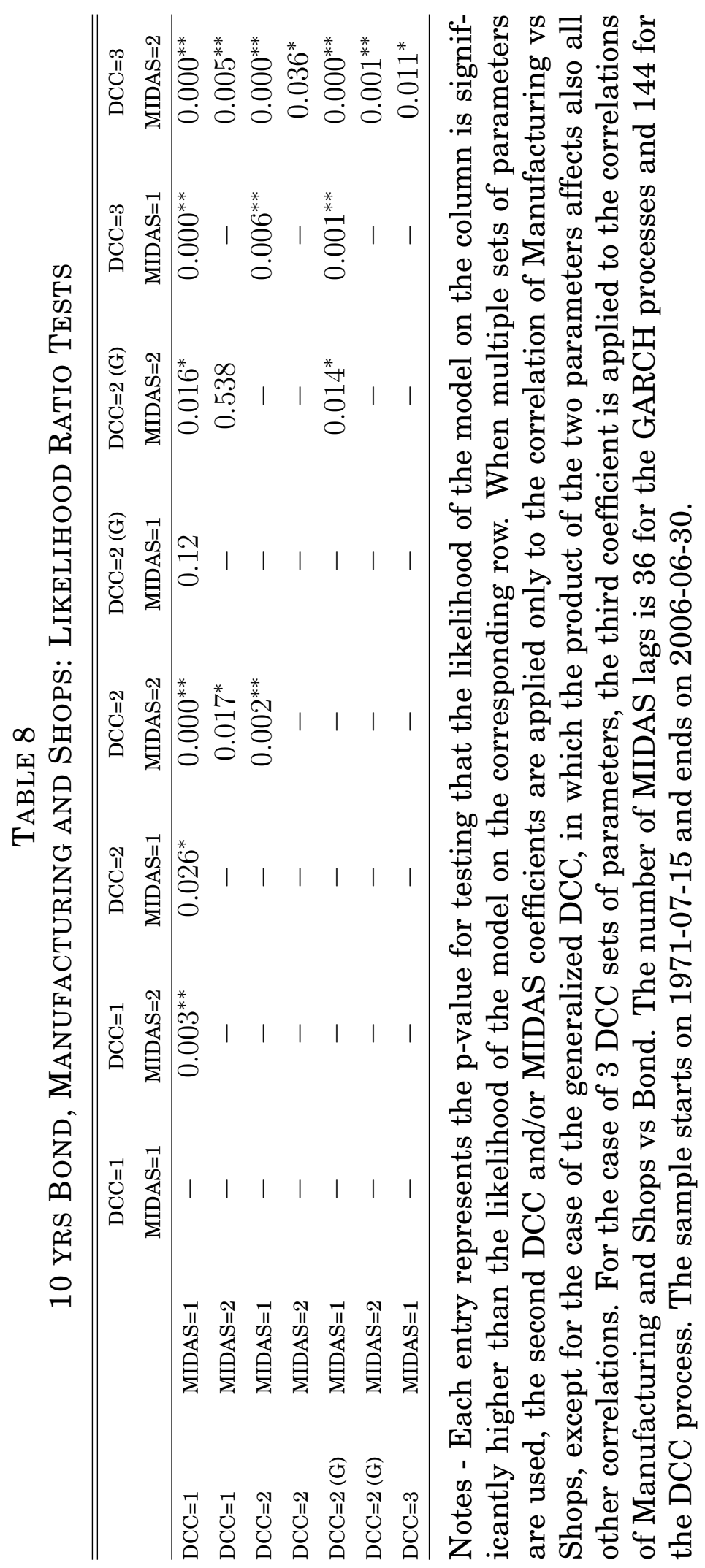



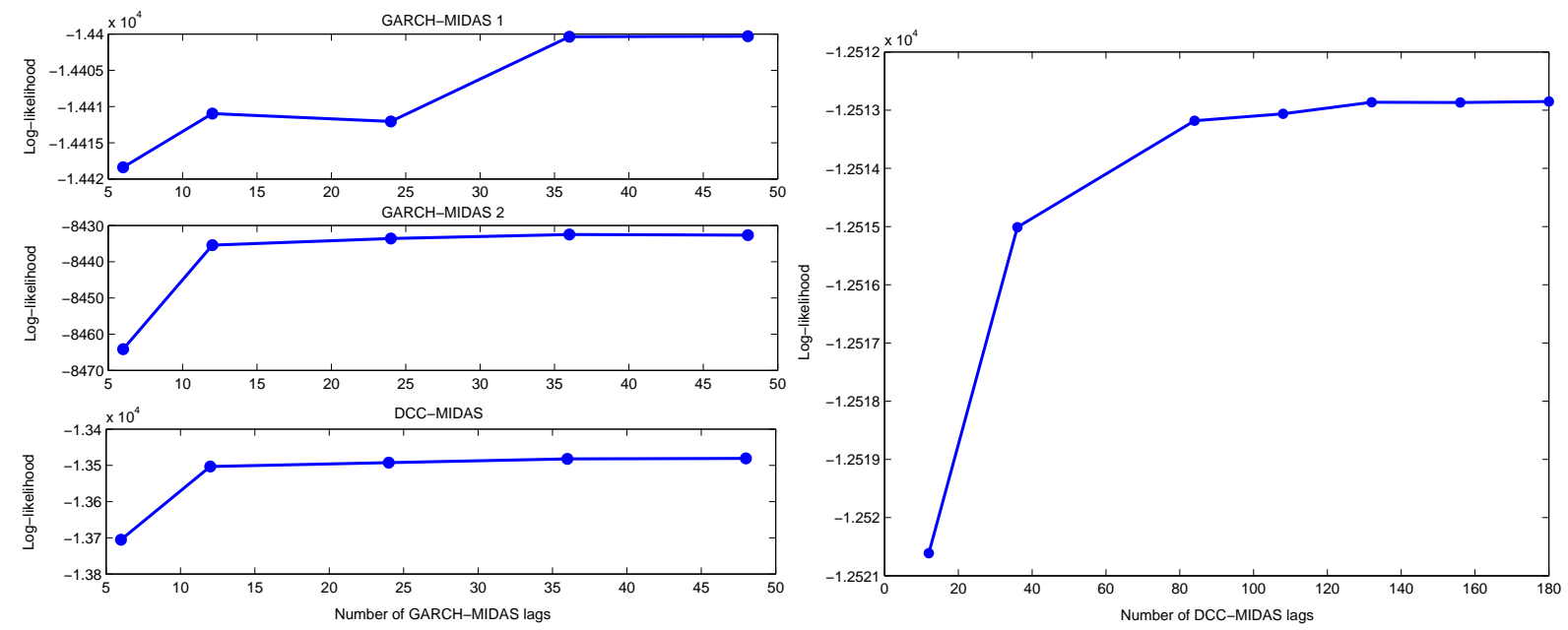

FIG. 1 - Log-likelihoods of variances and correlations for increasing number of MIDAS lags. The left panel reports the log-likelihoods obtained by changing the number of GARCH-MIDAS lags for the energy portfolio and the 10 year bond variance and the correlation estimator, respectively. The right panel shows the log-likelihood of the correlation estimator for increasing DCC-MIDAS lags. 

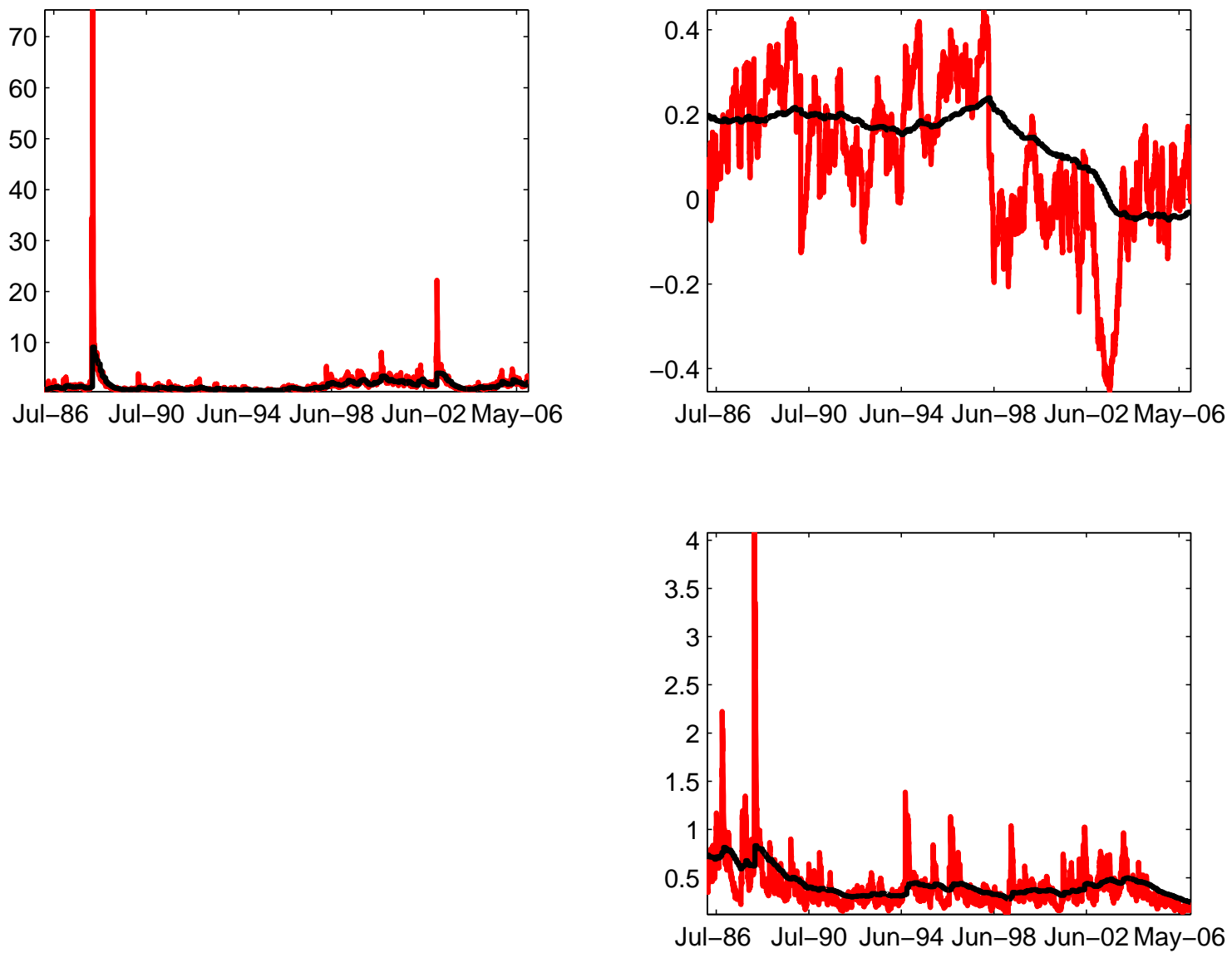

FIG. 2 - Long and short run volatilities and correlations for the energy portfolio and the 10 year bond. The pictures on the main diagonal refer to conditional variances of the energy portfolio and of the 10 year bond and the one on the off diagonal reports conditional correlations. In each panel the dark line refers to the long run and the light line represents the short run. 


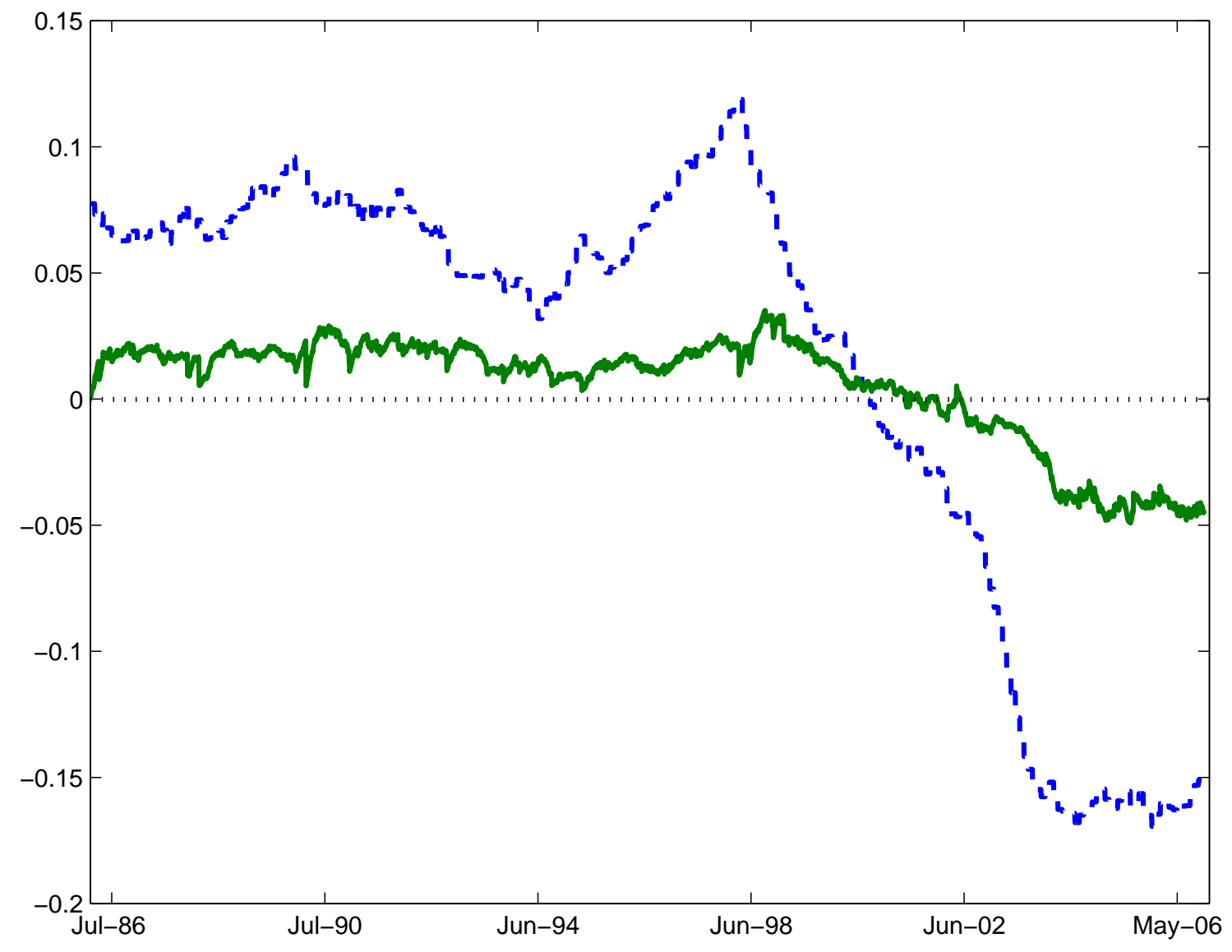

FIG. 3 - Differences in short run dynamics. The solid line represents the difference of short run correlations computed according to the DCC-MIDAS and the original DCC. The dashed line represent the excess long run correlation obtained as the difference between the DCC-MIDAS and the unconditional correlation. 

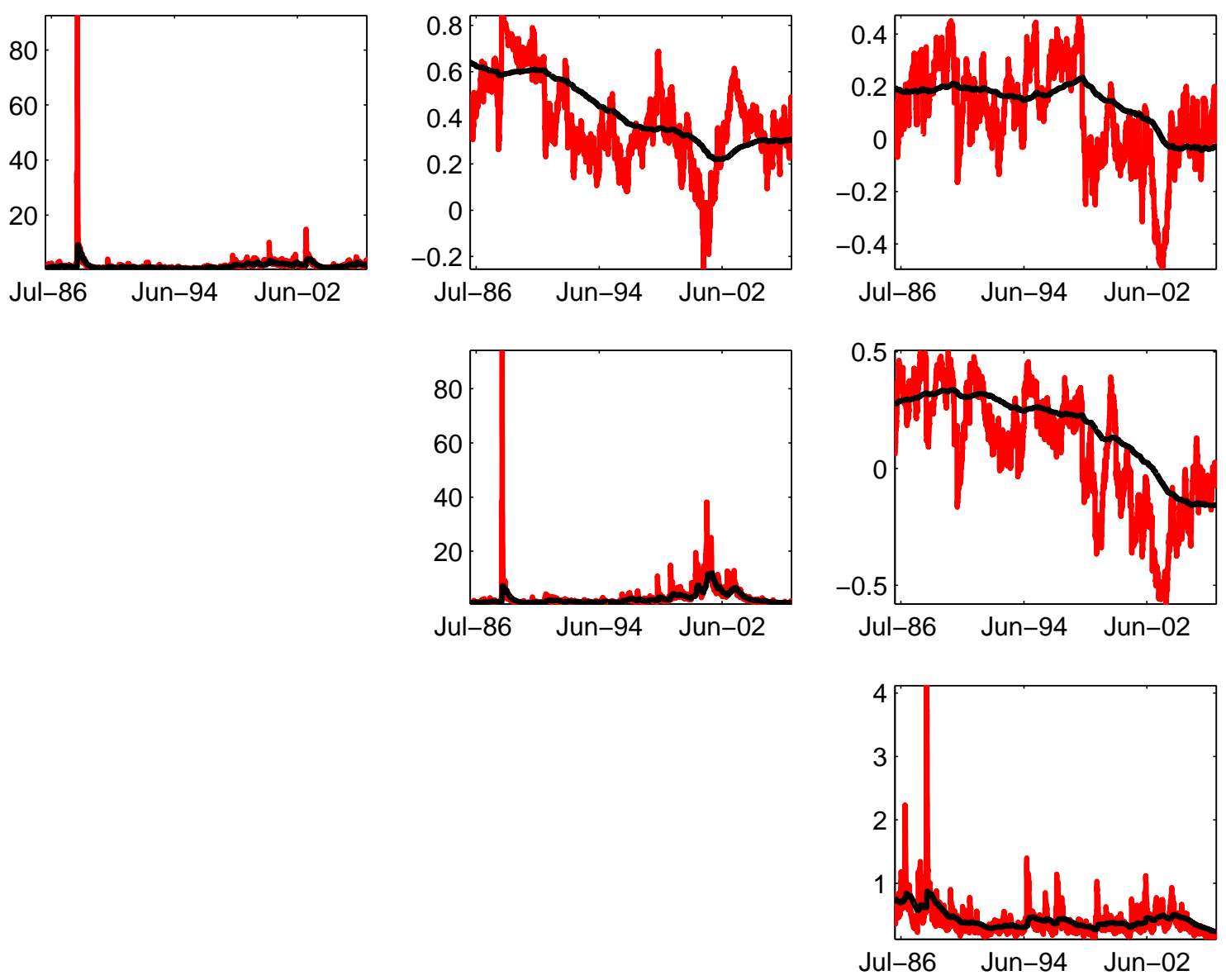

FIG. 4 - Long and short run volatilities and correlations for the energy and hi-tech portfolios and the 10 year bond. The pictures on the main diagonal refer to conditional variances of energy and hi-tech portfolios and of 10 year bond and those on the off diagonal report conditional correlations among the same group of asset returns. In each panel the dark line refers to the long run and the light line represents the short run. 

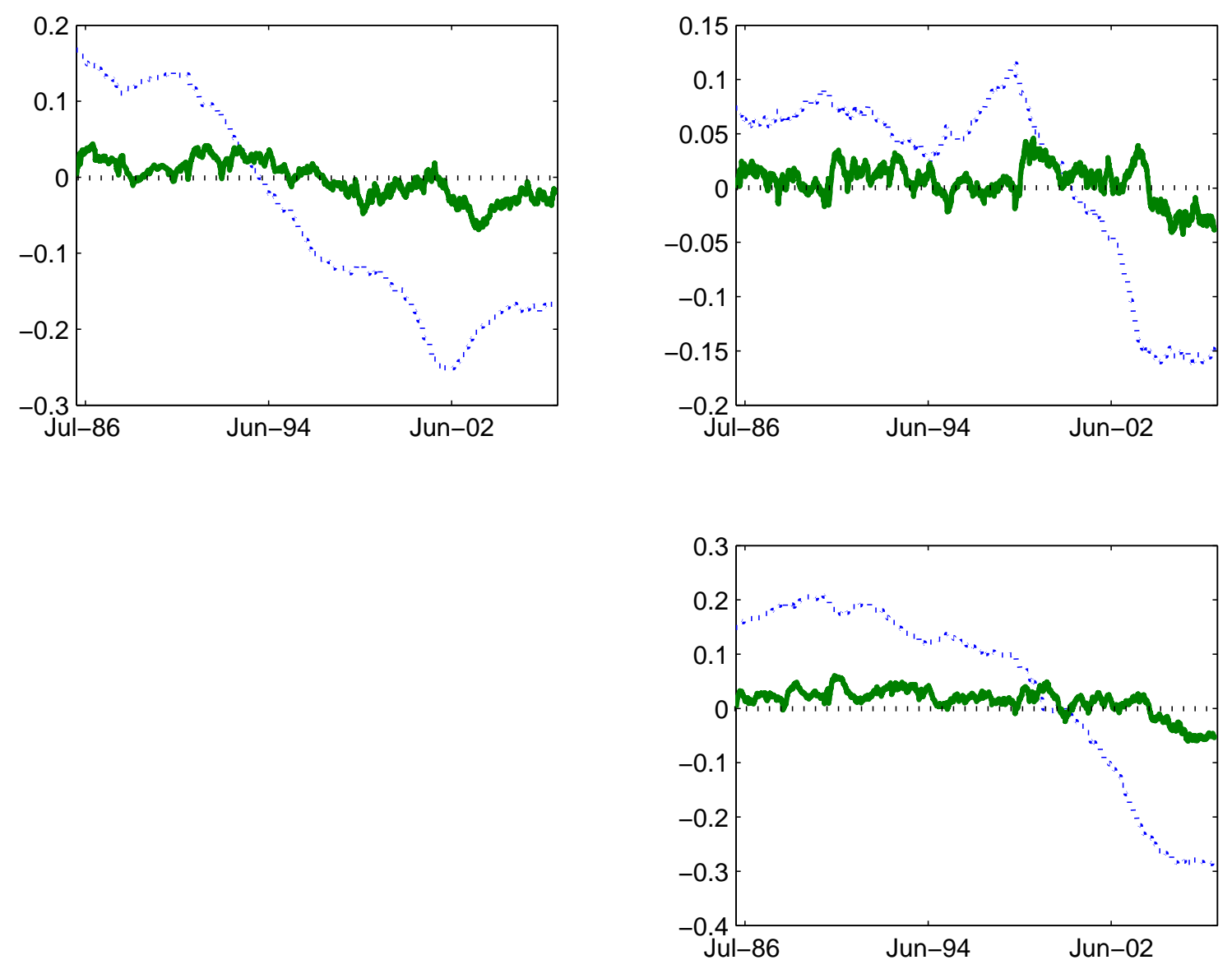

FIG. 5 - Differences in short run dynamics. In each subplot, the solid line represents the difference of short run correlations computed according to the DCC-MIDAS and the original DCC. The dashed line represent the excess long run correlation obtained as the difference between the DCC-MIDAS and the unconditional correlation. Starting from the top-left corner, the three subplots refer to the correlation of energy and hi-tech portfolios, energy portfolio and 10 year bond and hi-tech portfolio and 10 year bond. 

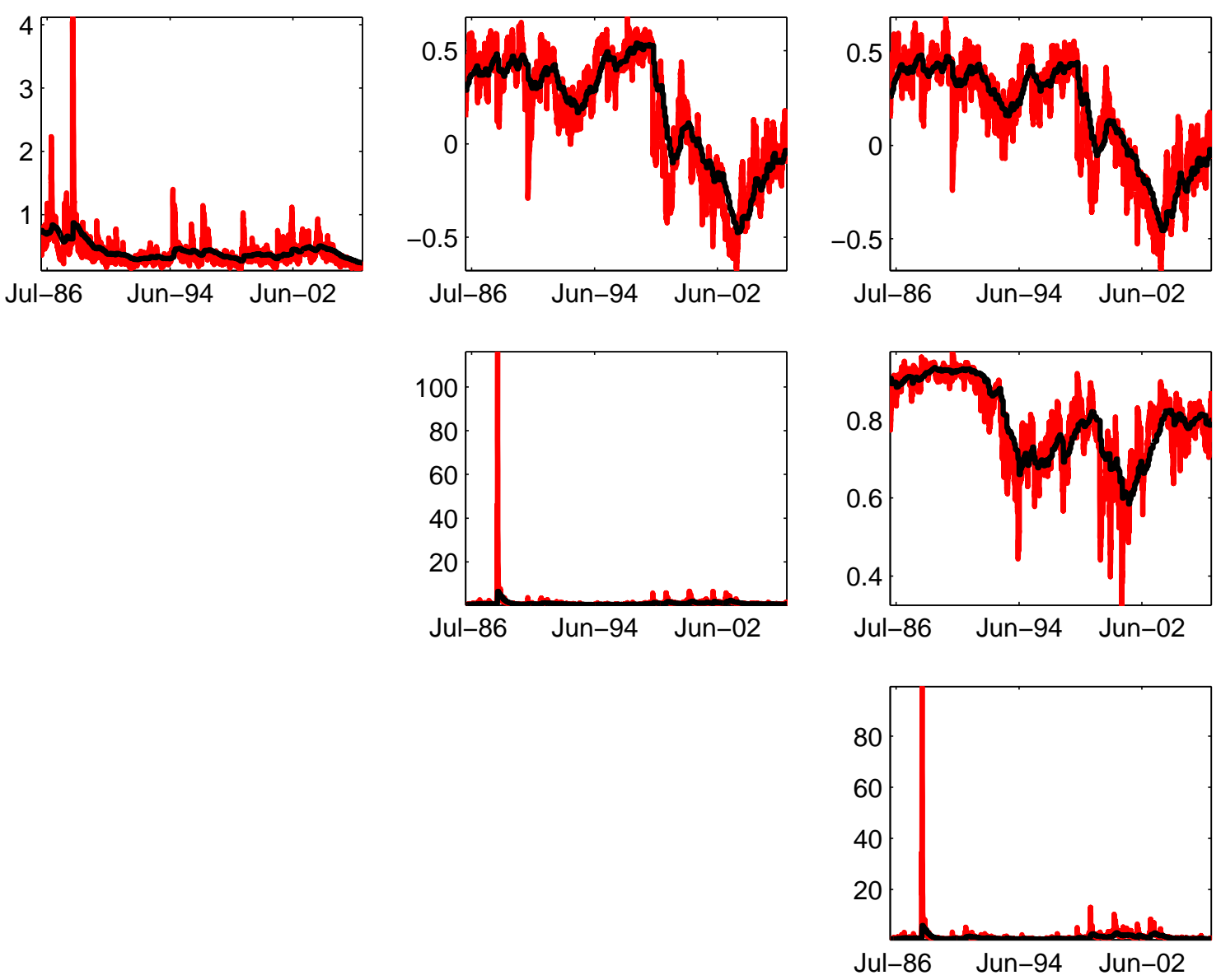

FIG. 6 - Long and short run volatilities and correlations for the 10 year bond and Manufacturing and Shops portfolios. The pictures on the main diagonal refer to conditional variances of bond, manufacturing and shops and those on the off diagonal report conditional correlations among the same group of asset returns. In each panel the dark line refers to the long run and the light line represents the short run. 

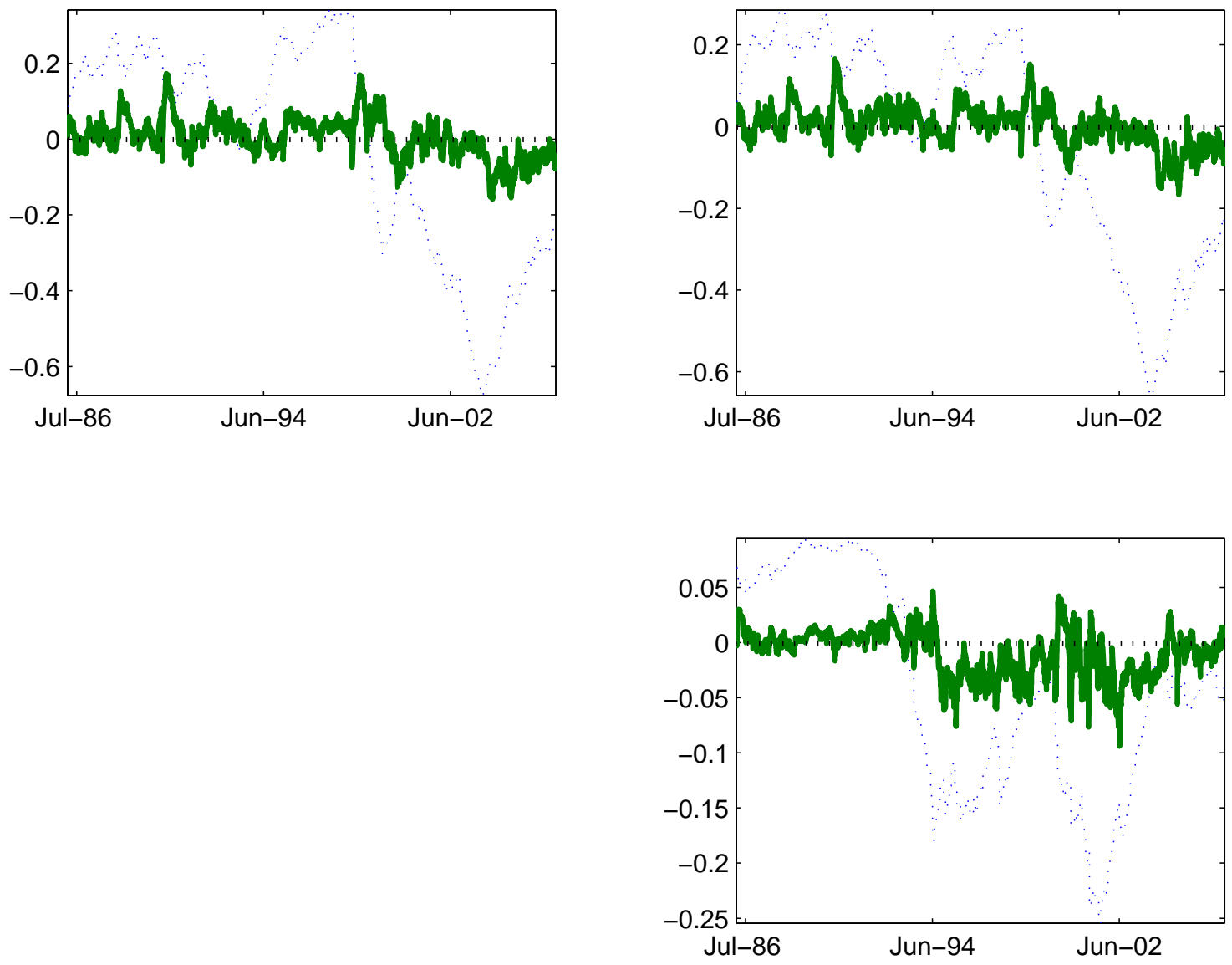

FIG. 7 - Differences in short run dynamics. In each subplot, the solid line represents the difference of short run correlations computed according to the DCC-MIDAS and the original DCC. The dashed line represent the excess long run correlation obtained as the difference between the DCC-MIDAS and the unconditional correlation. Starting from the top-left corner, the three subplots refer to the correlation of bond and manufacturing portfolio, bond and shops portfolio and manufacturing and shops portfolios. 

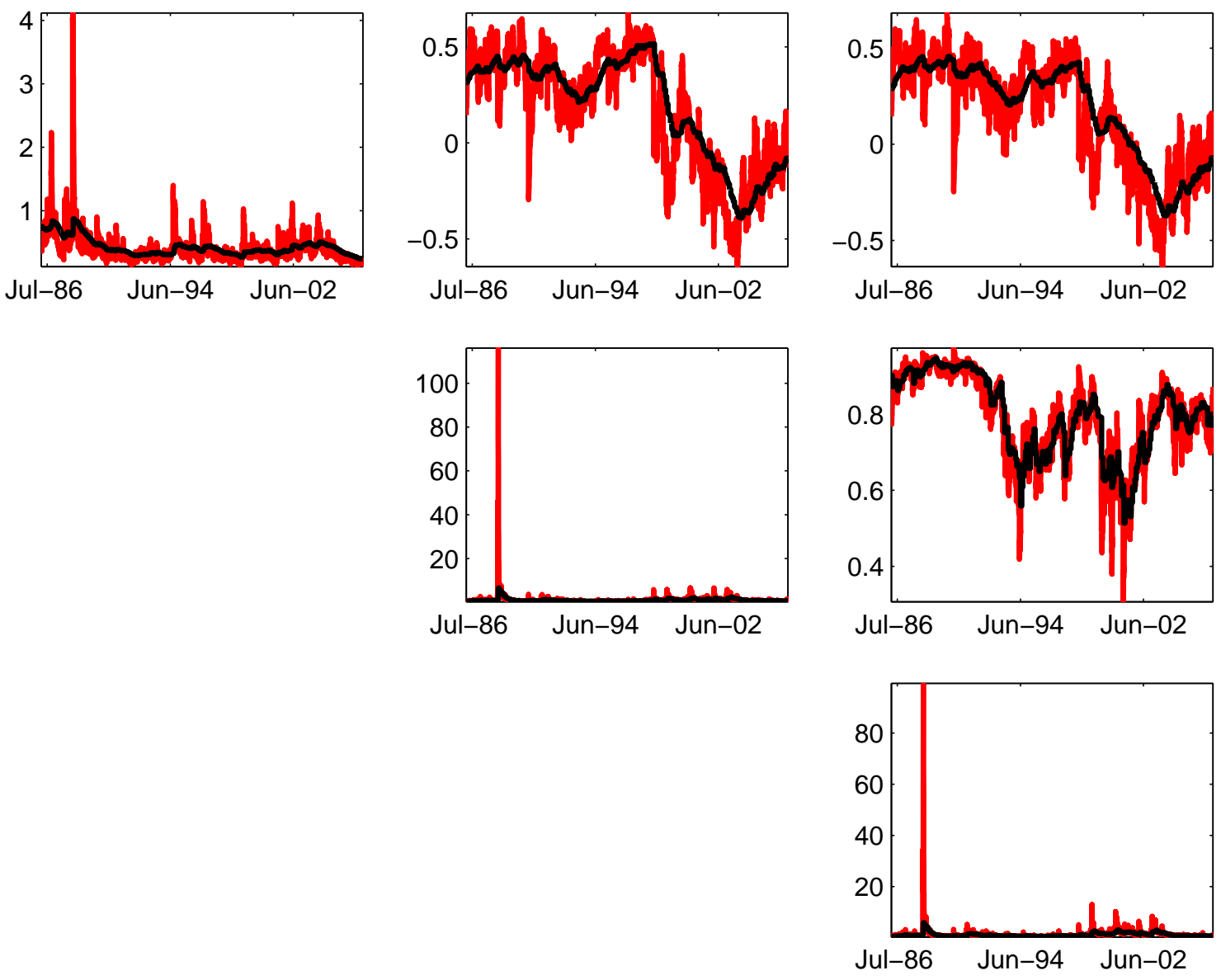

FIG. 8 - Long and short run volatilities and correlations for the 10 year bond and Manufacturing and Shops portfolios with 2 MIDAS filters. The second MIDAS filter is applied to the correlation between the manufacturing and the shops portfolios. The pictures on the main diagonal refer to conditional variances of bond, manufacturing and shops and those on the off diagonal report conditional correlations among the same group of asset returns. In each panel the dark line refers to the long run and the light line represents the short run. 

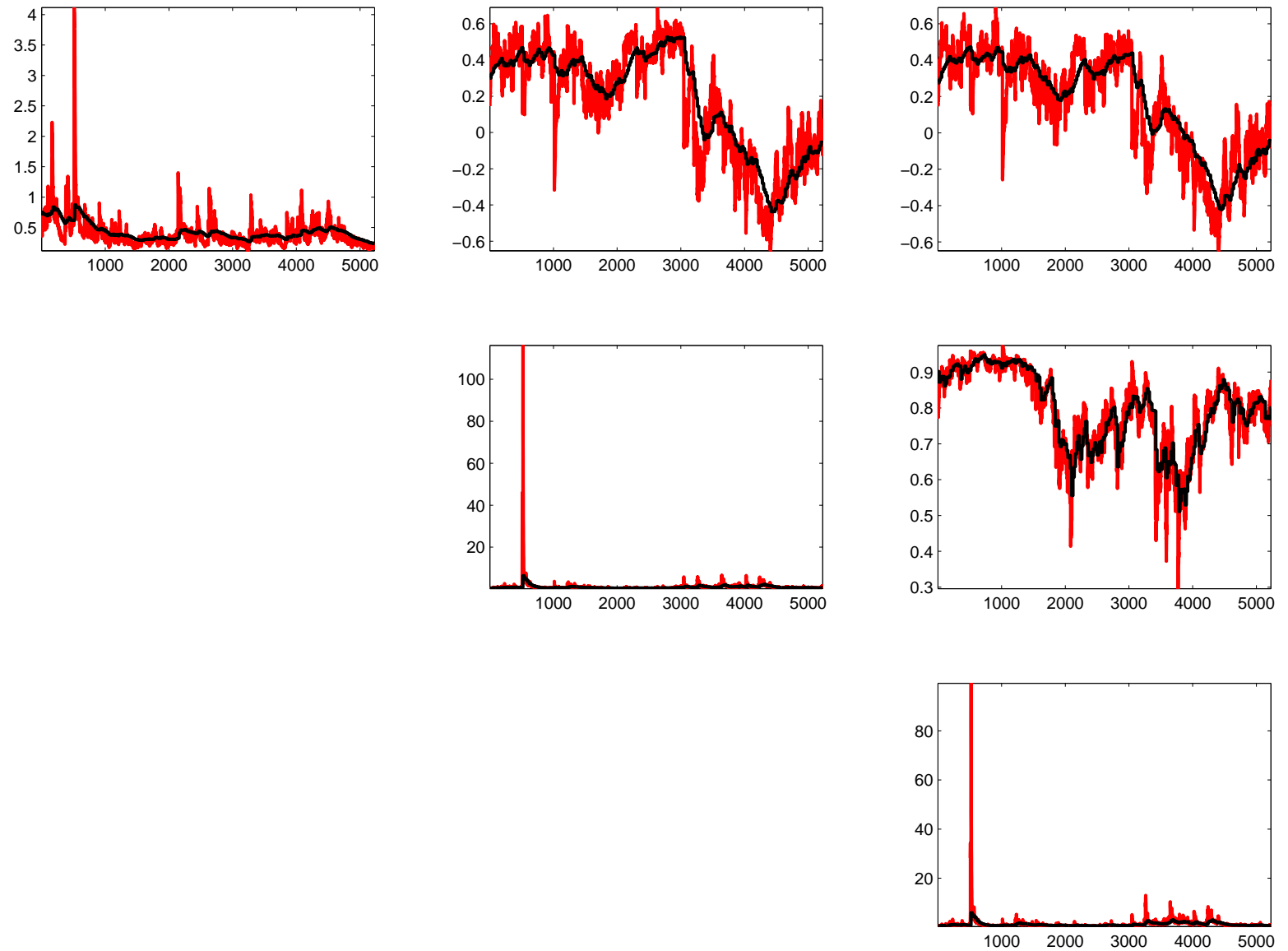

Fig. 9 - Long and short run volatilities and correlations for the 10 year bond and Manufacturing and Shops portfolios using the Generalized DCC-MIDAS with 2 DCC set of parameters and 2 MIDAS filters. The second set of parameters is applied to the correlation between the manufacturing and the shops portfolios. The pictures on the main diagonal refer to conditional variances of bond, manufacturing and shops and those on the off diagonal report conditional correlations among the same group of asset returns. In each panel the dark line refers to the long run and the light line represents the short run. 